\title{
Domain Decomposition Method for Harmonic Wave Propagation : A General Presentation
}

Francis Collino, Souad Ghanemi, Patrick Joly

$N^{\circ} 3473$

Août 1998

THÈME 4 



\title{
Domain Decomposition Method for Harmonic Wave Propagation : A General Presentation
}

\author{
Francis Collino, Souad Ghanemi, Patrick Joly \\ Thème 4 - Simulation et optimisation \\ de systèmes complexes \\ Projet Ondes
}

Rapport de recherche $\mathrm{n}^{\circ} 3473$ - Aout 1998 - 53 pages

\begin{abstract}
In this paper we give a general presentation of non overlapping domain decomposition methods for harmonic wave propagation models. Our abstract framework lead to concise convergence proofs and contains some recent developments about the use of non local transmission conditions. It also includes applications to acoustic, electromagnetic or elastic waves, as well as the treatment of space discretization.
\end{abstract}

Key-words: Helmholtz equation, Domain Decomposition Method, Maxwell, elastodynamic, non local operators 


\section{Méthode de décomposition de domaine pour la propagation des ondes harmoniques : une présentation générale}

Résumé : Nous décrivons une présentation générale des méthodes de décomposition de domaines pour des modèles de propagation d'ondes harmoniques. Notre cadre abstrait conduit à des démonstrations de convergences concises et contient des développements récents sur l'utilisation de conditions de transmission non locales aux interfaces. Il peut s'appliquer aux équations des ondes acoustiques, élastiques ou électromagnétiques aussi bien dans leur version continue que discrétisée.

Mots-clé : Décomposition de domaines, équation d'Helmoltz, équations de Maxwell, opérateurs non locaux 


\section{Contents}

1 Introduction. Presentation of the paper $\quad 3$

2 A model problem - Reformulation at interfaces $\quad 6$

2.1 Description of the model problem . . . . . . . . . . . . . 6

2.2 An equivalent transmission problem . . . . . . . . . . . 7

2.3 Some operators . . . . . . . . . . . . . . . . . . . . . . . . . . . . . . . .

2.4 Reformulation of the transmission problem at interfaces . . . . . . . 11

3 The iterative domain decomposition algorithms 12

3.1 The basic iterative algorithms and its interpretations . . . . . . . . . 12

3.2 Two variants of the basic algorithm using local transmission problems . . . 15

3.2.1 The relaxed Jacobi's algorithm . . . . . . . . . . . 15

3.2.2 The Gauss Seidel Algorithm . . . . . . . . . . . . 16

3.3 General transmission operators . . . . . . . . . . . . 18

3.3.1 Introduction . . . . . . . . . . . . . . . 18

3.3.2 Well-posedness of local problems . . . . . . . . . . . 19

3.3.3 Reformulation at interfaces . . . . . . . . . . . . . . . 21

3.3.4 The Domain Decomposition algorithms . . . . . . . . . . 23

4 Convergence analysis $\quad 24$

4.1 A first general result . . . . . . . . . . . . . . . . . 25

4.2 A case of exponential convergence . . . . . . . . . . . . . 27

5 Determination of the rate of convergence in a simple case 32

5.1 Presentation of the example, notations. . . . . . . . . . . 32

5.2 A general error estimate . . . . . . . . . . . . . . . . . 34

5.3 Analysis of the non zero eigenvalues of $A \ldots \ldots 36$

5.4 The convergence rate for the Jacobi's algorithm . . . . . . . . . . 37

5.5 The convergence rate for the Gauss Seidel's algorithm . . . . . . . . . . 39

6 Other applications $\quad \mathbf{4 0}$

6.1 Space discretization and domain decomposition . . . . . . . . . . 41

6.2 Other physical models . . . . . . . . . . . . . . . . 44

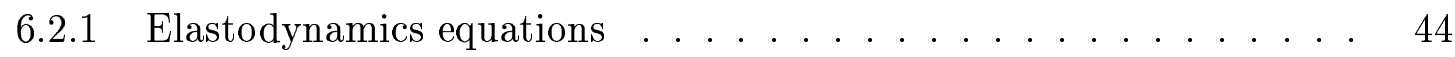

6.2.2 3D Maxwell's system .................... 45

$\begin{array}{lll}7 & \text { Numerical results } & \mathbf{4 7}\end{array}$

7.1 An illustration of the interest of non local operators . . . . . . . . . . . 47

7.2 A new choice : transparent like operators . . . . . . . . . . . . 49

7.3 On the influence of the geometry of the partition of $\Omega \ldots \ldots$ 


\section{Introduction. Presentation of the paper}

Time harmonic wave propagation phenomena appear to be a privileged field of application of domain decomposition methods (DDM): indeed, the solution is in general highly oscillatory (except may be for very low frequency computations) and its approximation requires generally a very thin space discretization, leading to large size linear system which can not reasonably be solved using direct factorization methods. Moreover, these systems are complex and non hermitian (even if they are symmetric in general), so that usual iterative methods either do not converge or converge only slowly, without any theoretical guarantees of convergence, [8]. In this context, it is natural to try to reduce the solution of the full problem to a succession of smaller local problems that could be handled for instance by direct methods. This is precisely the purpose of the domain decomposition techniques. However, the solution of the Helmholtz type equations which describe wave propagation in the frequency domain has not the same nice properties than the solutions of more standard elliptic equations such as Laplace equation: it does not naturally correspond to the minimum of a quadratic functional, the maximum principle does not hold, ... That is why the ideas of the famous overlapping Schwartz algorithm ([25], [26], [27]) can not be applied to harmonic wave propagation models. This probably explains why the research on DDM for such models started only recently. The objective of the present paper is to give a survey of some works in this field made by the authors and some of their colleagues and to present some more recent developments.

The methods we are going to speak about are always non overlapping iterative methods : the domains only communicate by their interfaces. As usual all the difficulty lies in the way one makes the different sub-domains communicate between each others : the key point are thus the choice of good transmission conditions and the choice of a clever iterative scheme. The starting point of the methods is the choice of suitable transmission conditions which involve an appropriate linear combination of Neumann and Dirichlet data with imaginary coefficients. To indicate the basic ideas, let us consider the very simple example of the 1D Helmholtz equation.

$$
\left.-\partial_{x x}^{2} u-\omega^{2} u=f, \quad x \in\right]-a, a[,
$$

with transparent boundary conditions at $x= \pm a$,

$$
\left(\partial_{x} u \pm i \omega u\right)( \pm a)=0 \text {. }
$$

Let us consider a partition of the interval ] $-a, a[$ into sub-intervals $] x_{k}, x_{k+1}\left[, x_{0}=-a<\right.$ $x_{1}<\ldots<x_{K}=+a$. Denoting by $u_{k+\frac{1}{2}}$ the restriction of $u$ to $] x_{k}, x_{k+1}[$, we have

$$
\left.-\partial_{x x}^{2} u_{k+\frac{1}{2}}-\omega^{2} u_{k+\frac{1}{2}}=f, \quad x \in\right] x_{k}, x_{k+1}[
$$

with the transmission conditions

$$
\left\{\begin{array}{l}
u_{k+\frac{1}{2}}\left(x_{k}\right)=u_{k-\frac{1}{2}}\left(x_{k}\right), \quad 0<k<K-1 \\
\partial_{x} u_{k+\frac{1}{2}}\left(x_{k}\right)=\partial_{x} u_{k-\frac{1}{2}}\left(x_{k}\right), \quad 0<k<K-1,
\end{array}\right.
$$


and the boundary conditions

$$
\begin{cases}\partial_{x} u_{\frac{1}{2}}\left(x_{0}\right)-i \omega u_{\frac{1}{2}}\left(x_{0}\right) & =0 \\ \partial_{x} u_{K-\frac{1}{2}}\left(x_{K}\right)+i \omega u_{K-\frac{1}{2}}\left(x_{K}\right) & =0 .\end{cases}
$$

The idea of the basic method, due to Despres [14], [13], [15], [17] and independently to [30] consists in rewriting (5) in the following form : for $k=1, \ldots K-1$

$$
\left\{\begin{array}{l}
\partial_{x} u_{k+\frac{1}{2}}\left(x_{k}\right)+i \omega u_{k+\frac{1}{2}}\left(x_{k}\right)=\partial_{x} u_{k-\frac{1}{2}}\left(x_{k}\right)+i \omega u_{k-\frac{1}{2}}\left(x_{k}\right) \\
-\partial_{x} u_{k+\frac{1}{2}}\left(x_{k}\right)+i \omega u_{k+\frac{1}{2}}\left(x_{k}\right)=-\partial_{x} u_{k-\frac{1}{2}}\left(x_{k}\right)+i \omega u_{k-\frac{1}{2}}\left(x_{k}\right) .
\end{array}\right.
$$

Therefore, looking at a single domain ] $x_{k}, x_{k+1}$ [, we have in particular, by picking one of the two equations 6 at $x=x_{k}$ and $x=x_{k+1}$

$$
\left\{\begin{array}{lc}
-\partial_{x x}^{2} u_{k+\frac{1}{2}}-\omega^{2} u_{k+\frac{1}{2}}=f, & x \in] x_{k}, x_{k+1}[ \\
-\partial_{x} u_{k+\frac{1}{2}}\left(x_{k}\right)+i \omega u_{k+\frac{1}{2}}\left(x_{k}\right)= & -\partial_{x} u_{k-\frac{1}{2}}\left(x_{k}\right)+i \omega u_{k-\frac{1}{2}}\left(x_{k}\right) \\
\partial_{x} u_{k+\frac{1}{2}}\left(x_{k+1}\right)+i \omega u_{k+\frac{1}{2}}\left(x_{k+1}\right)= & \partial_{x} u_{k+\frac{3}{2}}\left(x_{k+1}\right)+i \omega u_{k+\frac{3}{2}}\left(x_{k+1}\right) .
\end{array}\right.
$$

This system also holds for $k=0$ and $k=K-1$ if we use the convention $u_{-\frac{1}{2}}=u_{K+\frac{1}{2}}=0$. It can be seen as a problem which defines $u_{k+\frac{1}{2}}$, with both $u_{k-\frac{1}{2}}$ and $u_{k+\frac{3}{2}}$ considered to be known.

We then propose the following iterative procedure

$$
\left\{u_{k-\frac{1}{2}}^{n}\right\}_{k=1, K} \quad \longrightarrow \quad\left\{u_{k-\frac{1}{2}}^{n+1}\right\}_{k=1, K},
$$

where we have set

$$
\left\{\begin{array}{lc}
-\partial_{x x} u_{k+\frac{1}{2}}^{n+1}-\omega^{2} u_{k+\frac{1}{2}}^{n+1}=f, & x \in] x_{k}, x_{k+1}[ \\
-\partial_{x} u_{k+\frac{1}{2}}^{n+1}\left(x_{k}\right)+i \omega u_{k+\frac{1}{2}}^{n+1}\left(x_{k}\right)= & -\partial_{x} u_{k-\frac{1}{2}}^{n}\left(x_{k}\right)+i \omega u_{k-\frac{1}{2}}^{n}\left(x_{k}\right) \\
\partial_{x} u_{k+\frac{1}{2}}^{n+1}\left(x_{k+1}\right)+i \omega u_{k+\frac{1}{2}}^{n+1}\left(x_{k+1}\right)= & -\partial_{x} u_{k+\frac{3}{2}}^{n}\left(x_{k+1}\right)+i \omega u_{k+\frac{3}{2}}^{n}\left(x_{k+1}\right) .
\end{array}\right.
$$

It appears that this method is optimal in the sense that it converges exactly in a finite number of iterations. Indeed, introducing the error defined in each sub-domain by

$$
e_{k+\frac{1}{2}}^{n}=u_{k+\frac{1}{2}}-u_{k+\frac{1}{2}}^{n}
$$

We know that the $e_{k+\frac{1}{2}}^{n}$ satisfy (9) with $f=0$. Therefore we can write

$$
e_{k+\frac{1}{2}}^{n}=a_{k+\frac{1}{2}}^{n} \exp (i \omega x)+b_{k+\frac{1}{2}}^{n} \exp (-i \omega x) .
$$


Observing that

$$
\left\{\begin{array}{l}
\partial_{x} e_{k+\frac{1}{2}}^{n}+i \omega e_{k+\frac{1}{2}}^{n}=2 i \omega a_{k+\frac{1}{2}}^{n} \exp (i \omega x) \\
\partial_{x} e_{k+\frac{1}{2}}^{n}-i \omega e_{k+\frac{1}{2}}^{n}=-2 i \omega b_{k+\frac{1}{2}}^{n} \exp (-i \omega x)
\end{array}\right.
$$

the transmission conditions lead to

$$
\begin{aligned}
& a_{k-\frac{1}{2}}^{n+1}=a_{k+\frac{1}{2}}^{n}, \quad k=1,2, \ldots, K-1 \\
& b_{k+\frac{1}{2}}^{n+1}=b_{k-\frac{1}{2}}^{n}, \quad k=1,2, \ldots, K-1,
\end{aligned}
$$

while the boundary conditions express that

$$
a_{K-\frac{1}{2}}^{n}=0, \quad b_{\frac{1}{2}}^{n}=0, \quad \forall n
$$

From (13) and (14), one easily deduces that, after K iterations

$$
a_{k-\frac{1}{2}}^{K}=b_{k-\frac{1}{2}}^{K}=0, \quad k=1, \ldots, K
$$

which means that the process converges in $K$ iterations, where $K$ is the number of subdomains. In this sense, the iterative procedure we proposed based on the use of the mixed quantities quantities $\pm \frac{d u}{d x}+i \omega u$ is optimal.

The methods we are going to treat in the paper are essentially extensions and variants of this basic algorithm. The extensions mainly concern higher space dimensions and other type of equations. The variants concern the interface conditions and the iteration procedure. In this paper, we make a particular effort of formalisation and abstraction in order to give a unified presentation of all the extensions and variants. The outline is as follows.

In section 2, we present the model problem (the Helmholtz equation) that we consider in the major part of this article. The main objective of this section is to write an equivalent transmission problem on a partition of $\Omega$ using, as in $1 \mathrm{D}$, local transmission operators. We reformulate this transmission problem in terms of a new problem whose unknowns are quantities defined at the interfaces between the different sub-domains. We identify an abstract general form of this problem modulo the introduction of appropriate linear operators. In section 3, we describe the various iterative domain decomposition algorithms we have chosen to discuss. In particular, we introduce the notion of Jacobi and Gauss-Seidel algorithms as well as their relaxed version. In section 3.3, we introduce a more general class of algorithms based on the use of a more general class of transmission operators. We show they fit the general framework we have constructed in the previous section. Section 4 is devoted to the convergence analysis. Our proof is only based on a priori estimates. We emphasize a particular choice of partition of $\Omega$ and of transmission operators which guarantees an exponential rate of convergence. In section 5 , we analyze in a more quantitative way the speed of convergence of our algorithms in a very particular case of two domains. This permits us to discuss the choice of some of the parameters of the method. In section 6, we present various extensions within the abstract formalism They concern first the space discretization of the model problem then the application of our methods to 
other physically relevant problems. Finally, we illustrate some aspects of our theoretical analysis by some numerical results.

We cannot treat in a few pages all the aspects of DDM for harmonic wave propagation. Let us conclude this introduction by a rapid review of the literature (a useful complement to the present paper). Historically, we must refer to the pioneering works of $\mathrm{B}$. Despres about the Helmholtz equation with purely local transmission conditions [13],[14], for the theoretical aspects, [16], [17] for computational issues, treated also in [31]) and Shaidurov [30] (see also [18] for an extension to absorbing media and [19] for a more algebraic point of view). Benamou extended the ideas therein to treat optimal control problems related to harmonic wave propagation (see [2], [1] and [3] for a review). The case of non local transmission conditions is treated in [21] and [20], while in [6], [7], the use of second order tangential differential operators (an intermediate situation) is considered. Overlapping methods are introduced in [5]. For the case of 3D Mawxell's equations, we refer to [17] and [9].

\section{A model problem - Reformulation at interfaces}

\subsection{Description of the model problem}

Here, $\Omega$ denotes a bounded domain of $\mathbf{R}^{N}$ with boundary $\Gamma$ we assume to be sufficiently smooth (at least Lipschitz continuous). We consider the problem with complex valued unknown function $u$

$$
\left(P_{\Omega}^{f}\right)\left\{\begin{array}{l}
\text { Find } u \in H^{1}(\Omega), \text { such that } \\
-\Delta u-\omega^{2} u=f, \quad \text { in } \Omega \\
\partial_{\nu} u+i \omega u=0, \quad \text { on } \Gamma,
\end{array}\right.
$$

( $\nu$ denotes the unit outgoing normal vector to $\Gamma$ ). For instance, the boundary condition can be seen as an approximate radiation condition at finite distance (as an approximation of the propagation of a wave in $\mathbb{R}^{N}$ ). The right hand side is the source term and $\omega>0$ designates the frequency.

Problem $\left(P_{\Omega}^{f}\right)$ enters in a more general class of problems $(\mathcal{O}$ referring to a bounded domain of $\mathbb{R}^{n}$ )

$$
\left(P_{\mathcal{O}}^{f, g}\right)\left\{\begin{array}{l}
\text { Find } u \in H^{1}(\mathcal{O}), \text { such that } \\
-\Delta u-\omega^{2} u=f, \quad \text { in } \mathcal{O} \\
\partial_{\nu} u+i \omega u=g, \quad \text { on } \partial \mathcal{O} .
\end{array}\right.
$$

Let us recall the following classical result (for a proof, see [10] or [14] for instance).

Theorem 1 If $(f, g)$ belongs to $L^{2}(\mathcal{O}) \times L^{2}(\partial \mathcal{O}),\left(P_{\mathcal{O}}^{f, g}\right)$ admits a unique solution in $H^{1}(\mathcal{O})$ which satisfies $\Delta u \in L^{2}(\mathcal{O})$, and $\partial_{\nu} u \in L^{2}(\partial \mathcal{O})$. Moreover there exists a constant 
$C=C(\mathcal{O})>0$ such that

$$
\|u\|_{H^{1}(\mathcal{O})}+\left\|\partial_{\nu} u\right\|_{L^{2}(\partial \mathcal{O})} \leq C\left(\|f\|_{L^{2}(\mathcal{O})}+\|g\|_{L^{2}(\partial \mathcal{O})}\right) .
$$

Let us simply recall that the proof uses Fredholm's alternative which reduces the existence proof to a uniqueness result. For such a result, one first prove that if $f=g=0$, then $u=\partial_{\nu} u=0$ on $\partial \mathcal{O}$ which implies by the unique continuation principle , [11], that $u$ is zero in $\mathcal{O}$. This first step deeply uses the absorption term $i \omega u$ in the boundary condition.

Remark 1 The choice of the model problem $\left(P_{\mathcal{O}}^{f}\right)$ is related to the sake of simplicity of the exposition. However, the reader will easily convince himself that all what we are going to say can be extended without any difficulty to the problem (more realistic from the point of view of the applications)

$$
\left\{\begin{array}{l}
\text { Find } u \in H^{1}(\Omega), \text { such that } \\
-\operatorname{div}\left(\frac{1}{\mu} \vec{\nabla} u\right)-\epsilon \omega^{2} u=f, \quad \text { in } \Omega \\
\partial_{\nu} u+i \omega Z u=g, \quad \text { on } \partial \Omega,
\end{array}\right.
$$

where $\epsilon(x), \mu(x)$ are bounded with bounded inverse positive functions defined in $\Omega$ and $Z(x): \Gamma \rightarrow \mathbb{C}$ satisfies $\Re(Z) \geq 0$ and $\Re(Z) \geq \delta>0$ for $x \in \Gamma_{0}$ where $\Gamma_{0}$ is a subset of $\Gamma$ with non zero measure.

\subsection{An equivalent transmission problem}

Let us consider a partition of $\Omega$ into $K$ sub-domains $\Omega_{k}$

$$
\begin{aligned}
& \bar{\Omega}=\bigcup_{k=1}^{K} \bar{\Omega}_{k} \\
& \Omega_{k} \cap \Omega_{j}=\emptyset, \quad \text { for } j \neq k \\
& \Omega_{k} \text { is Lipschitz continuous. }
\end{aligned}
$$

In what follows, we shall distinguish two notations for $\partial \Omega_{k} \cap \partial \Omega_{j}$ (interface between $\Omega_{k}$ and $\Omega_{j}$.

$$
\begin{aligned}
& \Sigma_{k j}=\partial \Omega_{k} \cap \partial \Omega_{j} \quad \text { as a part of } \Omega_{k} \\
& \Sigma_{j k}=\partial \Omega_{k} \cap \partial \Omega_{j} \quad \text { as a part of } \Omega_{j} .
\end{aligned}
$$

This is useful for distinguishing the two traces on $\partial \Omega_{k} \cap \partial \Omega_{j}$ for a function which can be discontinuous across an interface.

Remark 2 Nevertheless, when the distinction will not be important, we shall use indifferently the notation $\Sigma_{k j}, \Sigma_{j k}$ or $\partial \Omega_{k} \cap \partial \Omega_{k}$ without mentionning it explicitely. 


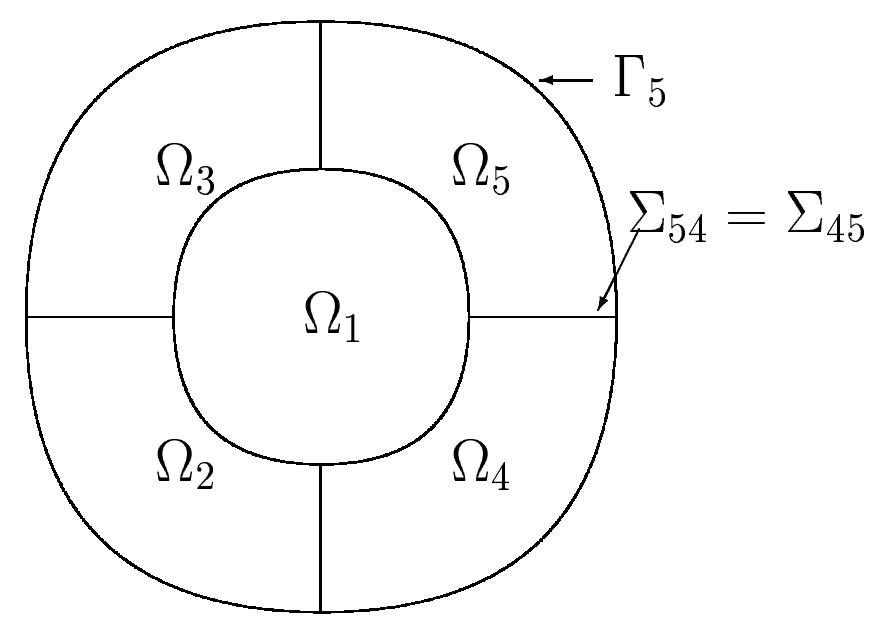

Figure 1: The domain $\Omega$ and its decomposition

Finally, we shall set

$$
\Sigma_{k k}=\partial \Omega \cap \partial \Omega_{k} .
$$

On $\partial \Omega_{k}$, we shall denote by $\nu_{k}$ the unit normal vector which is outgoing with respect to $\Omega_{k}$. Obviously, $\nu_{k}+\nu_{j}=0$ on $\Sigma_{k j}=\Sigma_{j k}$. Finally, if $u$ is a function of $L^{2}\left(\Omega_{k}\right)$, its restriction to $\Omega_{k}$ will be denoted by $u_{k}$. With this notation, it is clear that $u$ is solution of $\left(P_{\Omega}^{f}\right)$ if and only if $\left\{u_{k}, k=1, K\right\}$ is a solution of the transmission problem

$$
(\mathcal{P})_{t}\left\{\begin{array}{lc}
-\Delta u_{k}-\omega^{2} u_{k}=f_{k} & \text { in } \Omega_{k},
\end{array} \quad k=1, \ldots, K\right.
$$

The two transmission conditions can be rewritten in an equivalent way as $(k \neq j)$

$$
\begin{cases}\partial_{\nu_{k}} u_{k}+i \omega u_{k}=-\partial_{\nu_{j}} u_{j}+i \omega u_{j} & \text { on } \Sigma_{k j} \\ \partial_{\nu_{j}} u_{j}+i \omega u_{j}=-\partial_{\nu_{k}} u_{k}+i \omega u_{k} & \text { on } \Sigma_{j k}\end{cases}
$$

Therefore, $(\mathcal{P})_{t}$ can be seen as a collection of local problems of the form $\left(P_{\mathcal{O}}^{f, g}\right)$

$$
\left\{\begin{array}{lr}
-\Delta u_{k}-\omega^{2} u_{k}=f_{k} & \text { in } \Omega_{k} \\
\partial_{\nu_{k}} u_{k}+i \omega u_{k}=-\partial_{\nu_{j}} u_{j}+i \omega u_{j} & \text { on } \Sigma_{k j}, k \neq j \\
\partial_{\nu_{k}} u_{k}+i \omega u_{k}=0 & \text { on } \Sigma_{k k}
\end{array}\right.
$$




\subsection{Some operators}

Our goal in this section will be to reformulate the transmission problems as a problem whose unknown will be functions defined at the boundaries $\partial \Omega_{k}$, namely the traces

$$
x_{k}=\left(-\partial_{\nu_{k}} u_{k}+i \omega u_{k}\right)_{/ \partial \Omega_{k}} .
$$

For the validity of what follows, we shall be led to make the following regularity assumptions

$$
(\mathrm{H}): \quad x=\left(x_{k}\right) \in \prod_{k=1}^{K} V_{k} \quad V_{k}=L^{2}\left(\partial \Omega_{k}\right),
$$

which is not very restrictive in practice (Note that it is true if $\Omega_{k}$ is smooth enough).

Note that the space $V$ can also be decomposed according to the splitting of the interfaces $\partial \Omega_{k}$

$$
V=\prod_{j, k=1}^{K} V_{k j} \quad V_{k j}=L^{2}\left(\Sigma_{k j}\right)
$$

so that any element $y$ of $V$ can be written as

$$
y \equiv\left(y_{k}\right)_{k=1, K} \equiv\left(y_{k j}\right)_{k, j=1, K}
$$

In order to characterize $x$ as a solution of a linear equation in $V$, we are led to introduce some operators.

\section{Operators $E$ and $S$}

We first introduce:

$$
E \in \mathcal{L}\left(V ; \prod_{k=1}^{K} H^{1}\left(\Omega_{k}\right)\right)
$$

defined by

$$
e=\left(e_{k}\right)=E(y) \Leftrightarrow \mid \begin{aligned}
& -\Delta e_{k}-\Delta e_{k}=0, \quad \text { in } \Omega_{k} \\
& \partial_{\nu_{k}} e_{k}+i \omega e_{k}=y_{k}, \quad \text { on } \partial \Omega_{k}
\end{aligned}
$$

The fact that $E$ is well defined, linear and continuous is a direct consequence of Theorem 1. Note in particular that the boundary condition implies

$$
\partial_{\nu_{k}} e_{k} \in L^{2}\left(\partial \Omega_{k}\right), \quad k=1, \ldots K
$$

This permits us to define an other operator

$$
S \in \mathcal{L}(V ; V)
$$

by

$$
x=S(y) \quad \Leftrightarrow \quad x_{k}=-\partial_{\nu_{k}} e_{k}+i \omega e_{k}, \quad \text { where } e=E(x)
$$

A fundamental property of operator $S$ is the following one 
Lemma 1 The operator $S$ is unitary in $V$

Proof : The proof is classical. Let $(x, y)$ in $V \times V, u=E(x)$ and $v=E(y)$. By definition

$$
\begin{aligned}
& (x, y)=\sum_{k} \int_{\partial \Omega_{k}}\left(\partial_{\nu_{k}} u_{k}+i \omega u_{k}\right) \overline{\left(\partial_{\nu_{k}} v_{k}+i \omega v_{k}\right)} d \sigma \\
& (S x, S y)=\sum_{k} \int_{\partial \Omega_{k}}\left(-\partial_{\nu_{k}} u_{k}+i \omega u_{k}\right) \overline{\left(-\partial_{\nu_{k}} v_{k}+i \omega v_{k}\right)} d \sigma
\end{aligned}
$$

so that

$$
(S x, S y)-(x, y)=-2 i \omega \sum_{k} \int_{\partial \Omega_{k}}\left(\partial_{\nu_{k}} \bar{v}_{k} u_{k}-\partial_{\nu_{k}} u_{k} \bar{v}_{k}\right) d \sigma
$$

On the other hand by definition of $E$

$$
\begin{array}{ll}
\Delta u_{k}+\omega^{2} u_{k}=0 & (i) \\
-\Delta \bar{v}_{k}-\omega^{2} \bar{v}_{k}=0 & (i i) .
\end{array}
$$

We multiply (37)- $(i)$ by $\bar{v}_{k},(37)-(i i)$ by $u_{k}$, add the two equations and integrate over $\Omega_{k}$, we get

$$
\int_{\Omega_{k}}\left(\Delta u_{k} \bar{v}_{k}-u_{k} \Delta \bar{v}_{k}\right) d x=0 .
$$

Using Green's theorem, we obtain

$$
\int_{\partial \Omega_{k}}\left(\partial_{\nu_{k}} u_{k} \bar{v}_{k}-\partial_{\nu_{k}} \bar{v}_{k} u_{k}\right) d \sigma=0
$$

which proves, using (36), that

$$
\forall(x, y) \in V \times V, \quad(S x, S y)=(x, y) .
$$

and $S$ is unitary.

Remark 3 We have use the notation $S$ in relationship with the notations in Scattering theory, [23], [32] (tome 2, chapter 9 pages 145 to 240). In some sense, $S$ can be seen as a local scattering operator.

\section{The exchange operator $\Pi$ and its decomposition}

We introduce

$$
\Pi \in \mathcal{L}(V ; V)
$$

defined by

$$
z=\Pi y \Leftrightarrow \mid \begin{aligned}
& z_{k j} \equiv z_{k / \Sigma_{k j}}=y_{j / \Sigma_{j k}} \equiv y_{j k} k \neq j \\
& z_{k k} \equiv z_{k / \Sigma_{k k}}=0 .
\end{aligned}
$$

The main properties of the operator $\Pi$ are summarized in the following obvious lemma 
Lemma 2 The operator $\Pi$ is a symmetric contraction in $V$ and $\Pi^{2}=\Pi^{*} \Pi=P_{\Sigma}$ where $P_{\Sigma}$ is the orthogonal projector onto the subspace $V_{\Sigma}=\left\{x \in V, x_{/ \Gamma}=0\right\}$.

In the following, we will use the following decomposition of $\Pi$ (which depends of the numerotation of the subdomains)

$$
\Pi=\Pi^{+}+\Pi^{-},
$$

where

$$
z=\Pi^{+} y \Leftrightarrow \mid \begin{array}{ll}
z_{k j}=z_{k / \Sigma_{k j}}=y_{j / \Sigma_{j k}}=y_{j k}, & \forall k, j, k<j \\
z_{k j}=z_{k / \Sigma_{k j}}=0 & \forall k, j, k>j \\
z_{k}=z_{k / \Sigma_{k k}}=0 . &
\end{array}
$$

The following properties of $\Pi^{ \pm}$are straightforward

Lemma 3 The operators $\Pi^{ \pm}$are mutually adjoint and $P_{\Sigma^{+}}=\Pi^{+} \Pi^{-}$(resp. $\left.P_{\Sigma^{-}}=\Pi^{-} \Pi^{+}\right)$ is the orthogonal projector onto the subspace $V_{\Sigma}^{+}=\left\{x \in V, x_{/ \Sigma_{k j}}=0, \forall k>j\right\}$ (resp. $\left.V_{\Sigma}^{-}=\left\{x \in V, x_{/ \Sigma_{k j}}=0, \forall k<j\right\}\right)$.

Operator $A$ We introduce

$$
A=S \Pi \in \mathcal{L}(V, V)
$$

and we claim

Lemma 4 Operator $A$ is contractant in $V$ and $I-A$ is injective

Proof : Only the injectivity of $I-A$ needs to be clarified. It is in fact a consequence of the uniqueness result for $\left(P_{\mathcal{O}}^{f, g}\right)$. Indeed, setting $z=(I-A) x$ and $u=E(\Pi x)$, one easily computes that

$$
z_{k j}=\left(\partial_{\nu_{k}} u_{k}+\partial_{\nu_{j}} u_{j}\right)+i \omega\left(u_{k}-u_{j}\right) .
$$

If $z=0$, writing that $z_{k j}=z_{j k}=0$, we easily get that

$$
\begin{array}{ll}
\partial_{\nu_{k}} u_{k}+\partial_{\nu_{j}} u_{j}=0 & \text { on } \partial \Omega_{k} \cap \partial \Omega_{j} \\
u_{k}-u_{j}=0 & \text { on } \partial \Omega_{k} \cap \partial \Omega_{j},
\end{array}
$$

which means that $u$ is solution of $\Delta u+\omega^{2} u=0$ in $\Omega$. Writing that $z_{k k}=0$, we get $\partial_{\nu} u+i \omega u=0$ on each $\Sigma_{k k}$. Therefore $u=0$ and $x=0$.

\subsection{Reformulation of the transmission problem at interfaces}

Let us introduce $v_{k} \in H^{1}\left(\Omega_{k}\right)$ as the solution of the local problem

$$
\left\{\begin{array}{l}
-\Delta v_{k}-\omega^{2} v_{k}=f_{k} \\
\partial_{\nu_{k}} v_{k}+i \omega v_{k}=0 \text { on } \partial \Omega_{k},
\end{array}\right.
$$


and let us set

$$
g_{k}=-\partial_{\nu_{k}} v_{k}+i \omega v_{k / \partial \Omega_{k}}
$$

so that

$$
g=\left(g_{k}\right) \in V
$$

We have the

\section{Theorem 2}

(i) If $u=\left(u_{k}\right)$ is solution of $\mathcal{P}_{t}$, then $x \in V$ defined by (26) is solution of

$$
\left(\mathcal{P}_{g}\right): \quad(I-A) x=g .
$$

(ii) Reciprocally, if $x$ is solution of $\left(\mathcal{P}_{g}\right)$, then $u=v+E(\Pi x)$ is solution of $\mathcal{P}_{t}$

Proof : Let us set

$$
u_{k}=v_{k}+w_{k}
$$

As $\partial_{\nu_{k}} v_{k}+i \omega v_{k}=0$, we have

$$
\partial_{\nu_{k}} w_{k}+i \omega w_{k}=\partial_{\nu_{k}} u_{k}+i \omega u_{k}=\mid \begin{array}{ll}
-\partial_{\nu_{j}} u_{j}+i \omega u_{j} & \text { on } \Sigma_{k j} k \neq j \\
0 & \text { on } \Sigma_{k k} .
\end{array}
$$

Finally, $w_{k}$ satisfies to

$$
\left\{\begin{array}{l}
-\Delta w_{k}-\omega^{2} w_{k}=0 \\
\partial_{\nu_{k}} w_{k}+i \omega w_{k}=(\Pi x)_{k} \text { on } \partial \Omega_{k}
\end{array}\right.
$$

by definition of $E$, this implies $w=E(\Pi x)$ and

$$
-\partial_{\nu_{k}} w_{k}+i \omega w_{k}=S \Pi x=A x
$$

that is to say, using (26) and (49),

$$
x-g=A x
$$

This proves $(i)$. The proof of $(i i)$ is quite similar and will be omitted there.

\section{The iterative domain decomposition algorithms}

\subsection{The basic iterative algorithms and its interpretations}

We introduce in this section the algorithm initially proposed by Despres in [14], [12]. It consists in constructing a sequence

$$
u^{n}=\left(u_{k}^{n}\right) \in \prod_{k=1}^{K} H^{1}\left(\Omega_{k}\right)
$$


by the following process :

Assuming that $u^{n}$ is known and satisfies

$$
-\partial_{\nu_{k}} u_{k}^{n}+i \omega u_{k}^{n} \quad \in L^{2}\left(\partial \Omega_{k}\right), \quad k=1, \ldots, K,
$$

we define $u^{n+1}=\left(u_{k}^{n+1}\right)$, where $u_{k}^{n+1}$ is the solution of the non homogeneous boundary value problem

$$
\left\{\begin{array}{lr}
-\Delta u_{k}^{n+1}-\omega^{2} u_{k}^{n+1}=f, & \text { in } \Omega_{k} \\
\partial_{\nu_{k}} u_{k}^{n+1}+i \omega u_{k}^{n+1}=-\partial_{\nu_{j}} u_{j}^{n}+i \omega u_{j}^{n} & \text { on } \Sigma_{k j}, k \neq j \\
\partial_{\nu_{k}} u_{k}^{n+1}+i \omega u_{k}^{n}=0 & \text { on } \Sigma_{k k} .
\end{array}\right.
$$

If the initial value of $u_{k}^{0}$ is chosen such that $-\partial_{\nu_{k}} u_{k}^{0}+i \omega u_{k}^{0} \in L^{2}\left(\partial \Omega_{k}\right)$ (for instance if $\left.u_{k}^{0}=0, \forall k\right)$, it is clear via theorem 1 that this process is well posed in $\prod_{k} H^{1}\left(\Omega_{k}\right)$. Note also that at each step $n, u^{n}$ will be generally discontinuous across the various interfaces. However if $u^{n}$ converges in some sense to $u \in H^{1}\left(\Omega_{k}\right)$, then taking formally the limit in (59), we see that the function $u$ will satisfy to problem $\left(\mathcal{P}_{t}\right)$ equivalent to problem $\left(\mathcal{P}_{\Omega}^{f}\right)$.

It is now interesting to reinterpret algorithm (59) in terms of the formalism at interfaces introduced in the previous section. Let us set

$$
u_{k}^{n}=v_{k}+w_{k}^{n}
$$

we see that

$$
\left\{\begin{array}{lrl}
-\Delta w_{k}^{n+1}-\omega^{2} w_{k}^{n+1}=0, & \text { in } \Omega_{k} \\
\partial_{\nu_{k}} w_{k}^{n+1}+i \omega w_{k}^{n+1}=-\partial_{\nu_{j}} u_{j}^{n}+i \omega u_{j}^{n} & \text { on } \Sigma_{k j}, k \neq j \\
\partial_{\nu_{k}} w_{k}^{n+1}+i \omega w_{k}^{n+1}=0 & \text { on } \Sigma_{k k} .
\end{array}\right.
$$

If we set

$$
x_{k}^{n}=\left(-\partial_{\nu_{k}} u_{k}^{n}+i \omega u_{k}^{n}\right)_{/ \partial \Omega_{k}}=\left(-\partial_{\nu_{k}} w_{k}^{n}+i \omega w_{k}^{n}\right)_{/ \partial \Omega_{k}}+g_{k} .
$$

System (61) is equivalent to $w_{k}^{n+1}=E\left(\Pi x^{n}\right)$, hence, by definition of $S$

$$
x_{k}^{n+1}=\left(-\partial_{\nu_{k}} w_{k}^{n+1}+i \omega w_{k}^{n+1}\right)+g_{k}=\left(S \Pi x^{n}+g\right)_{k},
$$

or equivalently

$$
x^{n+1}=A x^{n}+g .
$$

One easily checks that, if $x^{0}=0$ for instance,

$$
x^{n}=\left(I+A+A^{2}+\ldots \ldots+A^{n}\right) g,
$$

so that the convergence of the process is formally linked to the convergence of the Neumann series

$$
(I-A)^{-1}=I+A+A^{2}+\ldots+A^{n}+\ldots
$$


Remark 4 The convergence proof shows that the convergence of the series (66) only holds in a weak sense as we will see later.

It is also instructive to reinterpret the algorithm in a matrix form in order to establish an analogy with usual iterative methods in linear algebra. In what follows we shall represent $x \in V$ as a column vector whose $k^{t h}$ component is $x_{k} \in L^{2}\left(\partial \Omega_{k}\right)$. With this representation, it is clear that the operators $S$ and $\Pi$ have the following block structure. $\left(\Pi_{j k}\right.$ is simply the multiplications by the characteristic function of $\Sigma_{k j}$ )

$$
\begin{array}{r}
\Pi=\left[\begin{array}{ccccc}
0 & \Pi_{12} & \Pi_{13} & \ldots & \Pi_{1 K} \\
\Pi_{21} & 0 & \Pi_{23} & & \Pi_{2 K} \\
\Pi_{31} & \Pi_{32} & \ddots & \ddots & \Pi_{3 K} \\
\vdots & & \ddots & \ddots & \Pi_{K-1 K} \\
\Pi_{K 1} & \ldots & \ldots & \Pi_{K K-1} & 0
\end{array}\right] \\
\Pi_{k j} \in \mathcal{L}\left(L^{2}\left(\partial \Omega_{k}\right), L^{2}\left(\partial \Omega_{j}\right)\right), \quad \Pi_{k j}^{*}=\Pi_{j k} \\
S=\left[\begin{array}{cccc}
S_{1} & 0 & 0 & 0 \\
0 & S_{2} & \ldots & 0 \\
\vdots & 0 & \ddots & \\
0 & & 0 & S_{K}
\end{array}\right], \quad S_{k} \in \mathcal{L}\left(L^{2}\left(\partial \Omega_{k}\right)\right),
\end{array}
$$

which illustrates that $S$ is purely local while $\Pi$ is an exchange operator. Performing the product $S \Pi$, we see that we can write (with $A_{i j}=S_{i} \Pi_{i j}$ )

$$
A=\left[\begin{array}{ccccc}
0 & A_{12} & A_{13} & \ldots & A_{1 K} \\
A_{21} & 0 & A_{23} & & A_{2 K} \\
A_{31} & A_{32} & \ddots & \ddots & A_{3 K} \\
\vdots & & \ddots & \ddots & \vdots \\
A_{K 1} & \ldots & \ldots & A_{K K-1} & 0
\end{array}\right]
$$

Thus, the operator to invert, $I-A$ is written

$$
I-A=\left[\begin{array}{ccccc}
I & -A_{12} & -A_{13} & \cdots & -A_{1 K} \\
-A_{21} & I & -A_{23} & & -A_{2 K} \\
-A_{31} & -A_{32} & \ddots & \ddots & -A_{3 K} \\
\vdots & & \ddots & \ddots & \vdots \\
-A_{K 1} & \ldots & \ldots & -A_{K K-1} & I
\end{array}\right]
$$

and can be splitted as the sum of its diagonal part, in fact the identity operator and its anti-diagonal part namely $-A$. In that sense, algorithm $x^{n+1}=A x^{n}+g$ is a Jacobi's algorithm. 


\subsection{Two variants of the basic algorithm using local transmission problems}

\subsubsection{The relaxed Jacobi's algorithm}

The idea is to modify the transmission condition (59) by replacing the in-going quantity $\partial_{\nu_{k}} u_{k}^{n+1}+i \omega u_{k}^{n+1}$ by a linear combination of the same quantity at steps $n$ and $n+1$. In other words, the local problem to be solved in $\Omega_{k}$ writes

$$
\left\{\begin{array}{cr}
-\Delta u_{k}^{n+1}-\omega^{2} u_{k}^{n+1}=f, & \\
\alpha\left(\partial_{\nu_{k}} u_{k}^{n+1}+i \omega u_{k}^{n+1}\right)+(1-\alpha)\left(\partial_{\nu_{k}} u_{k}^{n}+i \omega u_{k}^{n}\right)= & \text { in } \Omega_{k} \\
-\partial_{\nu_{j}} u_{j}^{n}+i \omega u_{j}^{n} & \text { on } \Sigma_{k j}, k \neq j \\
\partial_{\nu_{k}} u_{k}^{n+1}+i \omega u_{k}^{n+1}=0 & \text { on } \Sigma_{k k},
\end{array}\right.
$$

where $\alpha$ denotes some positive parameter. Note that one comes back to the original Jacobi's algorithm by taking $\alpha=1$. Note also that the local problem to be solved in each $\Omega_{k}$ is of type $\left(\mathcal{P}_{\mathcal{O}}^{f, g}\right)$, which ensures that the sequence $u^{n}=\left(u_{k}^{n}\right)$ is well defined in $H^{1}\left(\Omega_{k}\right)$. To reinterpret this new algorithm in terms of the formulation at interfaces, we introduce, as in section $3.2, w_{k}^{n}=u_{k}^{n}-v_{k}$ and

$$
\begin{aligned}
& x_{k}^{n}=\left(-\partial_{\nu_{k}} u_{k}^{n}+i \omega u_{k}^{n}\right)_{/ \partial \Omega_{k}}=\left(-\partial_{\nu_{k}} w_{k}^{n}+i \omega w_{k}^{n}\right)_{/ \partial \Omega_{k}}+g_{k} \\
& y_{k}^{n}=\left(\partial_{\nu_{k}} u_{k}^{n}+i \omega u_{k}^{n}\right)_{/ \partial \Omega_{k}}=\left(\partial_{\nu_{k}} w_{k}^{n}+i \omega w_{k}^{n}\right)_{/ \partial \Omega_{k}} .
\end{aligned}
$$

As $w_{k}^{n}$ satisfies $\omega^{2} w_{k}^{n}+\Delta w_{k}^{n}=0$, by definition of $S$, we get

$$
x^{n}-g=S y^{n}, \quad \text { for each } n .
$$

In addition, the boundary condition relating the $x^{n}$ and $y^{n}$ 's reads

$$
\alpha y^{n+1}+(1-\alpha) y^{n}=\Pi x^{n} .
$$

After multiplication by $S$, we eliminate $y^{n+1}$ and $y^{n}$. Setting $r=\frac{1}{\alpha}$, we obtain

$$
x^{n+1}=(1-r) x^{n}+r\left(A x^{n}+g\right),
$$

which is our relaxed Jacobi's algorithm. We shall see that for ensuring convergence we shall need to impose $r \in] 0,1]$. It corresponds to apply a fixed point method to the equation $\mathcal{P}_{g}$ rewritten in the form

$$
x=x-r((I-A) x-g) .
$$

A simple calculation, that we do not detail here, shows that $u^{n}$ can be reconstructed from $x^{n}$ by the formula

$$
u^{n+1}=(1-r) E\left(S^{*}\left(x^{n}-g\right)\right)+r E\left(\Pi x^{n}\right)+v,
$$


or equivalently $\left(S^{*} g=S^{*} x-\Pi x\right)$

$$
u^{n}=(1-r) E\left(S^{*}\left(x^{n}-x\right)\right)+(1-r) E(\Pi x)+r E\left(\Pi x^{n}\right)+v,
$$

identity which is clearly consistent with the limit equation

$$
u=E(\Pi x)+v
$$

Equation (80) suggests us another candidate as an approximation of $u$

$$
\tilde{u}^{n}=E\left(\Pi x^{n}\right)+v \text {. }
$$

One easily shows that $u^{n}$ and $\tilde{u}^{n}$ are related by

$$
u^{n+1}=(1-r) u^{n}+r \tilde{u}^{n}
$$

which shows that $\tilde{u}^{n}$ converges if and only if $u^{n}$ converges. It is thus useful to indicate how the sequence $\tilde{u}^{n}$ can be computed directly. In a first step, one solves

$$
\left\{\begin{array}{l}
-\Delta \tilde{u}_{k}^{n+1}-\omega^{2} \tilde{u}_{k}^{n+1}=f, \quad \text { in } \Omega_{k} \\
\partial_{\nu_{k}} \tilde{u}_{k}^{n+1}+i \omega \tilde{u}_{k}^{n+1}=x_{j k}^{n} \text { on } \Sigma_{k j}, k \neq j \\
\partial_{\nu_{k}} \tilde{u}_{k}^{n+1}+i \omega \tilde{u}_{k}^{n+1}=0 \text { on } \Sigma_{k k},
\end{array}\right.
$$

and, in a second step, the $x_{k j}$ are refreshed according to

$$
x_{k j}^{n+1}=(1-r) x_{k j}^{n}+r\left(-\partial_{\nu_{k}} \tilde{u}_{k}^{n+1}+i \omega \tilde{u}_{k}^{n+1}\right) .
$$

\subsubsection{The Gauss Seidel Algorithm}

This variant exploits the ordering of the domains and consists in taking profit of the subdomains $\Omega_{j}$ in which the solution at step $n+1$ has already been calculated. The relaxed version of this algorithm of this algorithm writes

$$
\left\{\begin{array}{l}
-\Delta u_{k}^{n+1}-\omega^{2} u_{k}^{n+1}=f, \quad \text { in } \Omega_{k} \\
\alpha\left(\partial_{\nu_{k}} u_{k}^{n+1}+i \omega u_{k}^{n+1}\right)+(1-\alpha)\left(\partial_{\nu_{k}} u_{k}^{n}+i \omega u_{k}^{n}\right)= \\
\quad-\partial_{\nu_{j}} u_{j}^{n+1}+i \omega u_{j}^{n+1} \text { on } \Sigma_{k j}, \quad j<k \\
\alpha\left(\partial_{\nu_{k}} u_{k}^{n+1}+i \omega u_{k}^{n+1}\right)+(1-\alpha)\left(\partial_{\nu_{k}} u_{k}^{n}+i \omega u_{k}^{n}\right)= \\
-\partial_{\nu_{j}} u_{j}^{n}+i \omega u_{j}^{n} \text { on } \Sigma_{k j}, \quad j>k \\
\partial_{\nu_{k}} u_{k}^{n+1}+i \omega u_{k}^{n+1}=0 \text { on } \Sigma_{k k} .
\end{array}\right.
$$


Once again each local problem to be solved is of type $\left(P_{\mathcal{O}}^{f, g}\right)$ so that the sequence $u^{n}=\left(u_{k}^{n}\right)$ is well defined in $\prod_{k} H^{1}\left(\Omega_{k}\right)$, provided that (27) holds and $-\partial_{\nu_{k}} u_{k}^{0}+i \omega u_{k}^{0} \in L^{2}\left(\partial \Omega_{k}\right)$ for each $k$.

In order to reinterpret this algorithm at interfaces, we use the decomposition $\Pi=$ $\Pi^{+}+\Pi^{-}$and simply remark that the transmission conditions on the $\Sigma_{k j}$ 's are equivalent to

$$
\alpha y^{n+1}+(1-\alpha) y^{n}=\Pi^{-} x^{n+1}+\Pi^{+} x^{n}+g .
$$

Setting once again $r=\frac{1}{\alpha}$ and after elimination of the $y^{n}$ 's, we obtain

$$
x^{n+1}=(1-r) x^{n}+r\left(S \Pi^{-} x^{n+1}+S \Pi^{+} x^{n}-g\right),
$$

which corresponds to a fixed point algorithm applied to the equation $\mathcal{P}_{g}$, rewritten as

$$
x-r S \Pi^{-} x=x-r\left(x-S \Pi^{+} x-g\right) .
$$

In the case $r=1$, we recover the non relaxed version Gauss-Seidel algorithm

$$
\left(I-A^{-}\right) x^{n+1}=A^{+} x^{n}+g,
$$

where $A^{-}=S \Pi^{-}, A^{+}=S \Pi^{+}$and $A=A^{+}+A^{-}$. Remark that, going back to the matrix interpretation of section 3.1 , the structure of the matrices $A^{+}, A^{-}$are of the form

$$
A^{+}=\left[\begin{array}{ccccc}
0 & A_{12} & A_{13} & \ldots & A_{1 K} \\
0 & 0 & A_{23} & & A_{2 K} \\
0 & 0 & \ddots & \ddots & A_{3 K} \\
\vdots & & \ddots & \ddots & \vdots \\
0 & \ldots & \ldots & 0 & 0
\end{array}\right], A^{-}=\left[\begin{array}{ccccc}
0 & 0 & 0 & \ldots & 0 \\
A_{21} & 0 & 0 & & 0 \\
A_{31} & A_{32} & \ddots & \ddots & 0 \\
\vdots & & \ddots & \ddots & \vdots \\
A_{K 1} & \ldots & \ldots & A_{K K-1} & 0
\end{array}\right]
$$

Matrix $A^{+}$is upper triangular while matrix $A^{-}$is lower triangular. This establishes a clear analogy between the usual Gauss-Seidel method in linear algebra.

In the same way of what has been done for the Jacobi's algorithm we can substitute to $u^{n}$ another relevement of $x^{n}$, let $\tilde{u}^{n}$, defined formally by (81). The explicit computation of the $x_{j k}^{n}$ and $\tilde{u}_{k}$, is given by (83) and (84) with $x_{j k}^{n}$ replaced by $x_{j k}^{n^{\star}}$ in the second line of (83), where $x_{j k}^{n^{\star}}=x_{j k}^{n}$ when $j>k$ and $x_{j k}^{n+1}$ when $j<i$.

Remark 5 As usual, the main drawback of the Gauss-Seidel algorithm is to be not parallelisable, contrary to the Jacobi's algorithm: the computation of the solution of each local subproblem must be solved accordingly to the ordering of the sub-domains. On the other hand, one may expect a better convergence. We shall come back on this point at section 5 . 


\subsection{General transmission operators}

\subsubsection{Introduction}

As we shall see later, the convergence of the previous algorithms is not exponential. This is due to the fact that operator $A$ admits a sequence of eigenvalues which converges to 1 in modulus (see section 5). The purpose of this section is to generalize the iterative algorithm in the hope to get an exponential rate of convergence. The basic idea is to replace the quantities $\partial_{\nu_{k}} u_{k / \Sigma_{k j}} \pm i \omega u_{k / \Sigma_{k j}}$ by quantities

$$
\partial_{\nu_{k}} u_{k / \Sigma_{k j}} \pm i \omega T_{j k}\left(u_{k / \Sigma_{k j}}\right)
$$

where $T_{k j}=T_{j k}$ are operators acting on functions defined on $\partial \Omega_{k} \cap \partial \Omega_{j}$, having appropriate properties that will be specified later. The more interesting case will be the one where $T_{j k}$ is some $L^{2}$ - symmetric pseudo-differential operator of order 1 . In that case both quantities $\partial_{\nu_{k}} u_{k}$ and $T_{j k} u_{k}$ belong to the same Sobolev space on $\partial \Omega_{k} \cap \partial \Omega_{j}$ so that the linear combinations (91) are "in some functional sense" more equilibrated than the quantities $\partial_{\nu_{k}} u_{k} \pm i \omega u_{k}$. We shall see in section 4 why such a property may lead to an exponential rate of convergence. Let us nevertheless remark that in practice this property will impose that the operators $T_{j k}$ will be non local. We shall come back to this point at the end of section 4.

In what follows, if $\Gamma$ denotes a Lipschitz-continuous manifold of dimension $n-1$, we shall adopt the notation $(\cdot, \cdot)_{L^{2}(\Gamma)}$ for the scalar product in $L^{2}(\Gamma)$ and $\langle\cdot, \cdot\rangle_{\Gamma}$ for the duality product between $H^{s}(\Gamma)$ and $H^{-s}(\Gamma)$ for $|s| \leq 1 / 2$. For each $\{j, k\}$ such that $\partial \Omega_{k} \cap \partial \Omega_{j}$ is not empty, we introduce an operator $T_{j k}$ satisfying

$$
\left\{\begin{array}{l}
T_{j k}=T_{k j} \in \mathcal{L}\left(H^{\frac{1}{2}}\left(\partial \Omega_{k} \cap \partial \Omega_{j}\right), H^{-\frac{1}{2}}\left(\partial \Omega_{k} \cap \partial \Omega_{j}\right)\right) \\
T_{j k} \text { is injective. }
\end{array}\right.
$$

The identity operator is an example of such an operator. Moreover, the injectivity of the operators $T_{j k}$ implies that our original problems $\left(P_{\Omega}^{f}\right)$ are equivalent to the following collection of local problems posed in $\Omega_{k}$ that we write formally

$$
\left\{\begin{array}{l}
-\Delta u_{k}-\omega^{2} u_{k}=f, \quad \text { in } \Omega_{k} \\
\partial_{\nu_{k}} u_{k}+i \omega T_{j k}\left(u_{k / \Sigma_{k j}}\right)=-\partial_{\nu_{j}} u_{j}+i \omega T_{j k}\left(u_{j / \Sigma_{j k}}\right) \quad \text { on } \Sigma_{k j}, k \neq j \\
\partial_{\nu_{k}} u_{k}+i \omega u_{k}=0 \quad \text { on } \Sigma_{k k} .
\end{array}\right.
$$

In order to ensure the well-posedness of each local problem (93), we shall be led to make the following assumption on $T_{j k}$, (which more or less expresses the fact that it is symmetric and positive)

$$
\begin{gathered}
\text { There exists } s \in\left[0, \frac{1}{2}\right] \text { and } \Lambda_{j k} \text { an isomorphism from } \\
H^{s}\left(\partial \Omega_{k} \cap \partial \Omega_{j}\right) \text { into } L^{2}\left(\partial \Omega_{k} \cap \partial \Omega_{j}\right) \text { such that : } \\
T_{j k}=\Lambda_{j k}^{*} \Lambda_{j k} .
\end{gathered}
$$


In (94), $s$ may depend on the operator $T_{j k}$, even it is not mentionned explicitly. The operator $\Lambda_{j k}^{*}$, adjoint of $\Lambda_{j k}$, is an isomorphism from $L^{2}\left(\partial \Omega_{k} \cap \partial \Omega_{j}\right)$ onto $H^{-s}\left(\partial \Omega_{k} \cap \partial \Omega_{j}\right)$. One deduces that $T_{j k}$ is a symmetric positive one to one operator from $H^{s}\left(\partial \Omega_{k} \cap \partial \Omega_{j}\right)$ onto $H^{-s}\left(\partial \Omega_{k} \cap \partial \Omega_{j}\right)$. We have

$$
\forall \varphi \in H^{\frac{1}{2}}\left(\Sigma_{k j}\right), \quad<T_{j k} \varphi, \varphi>_{\Sigma_{k j}}=\left\|\Lambda_{j k} \varphi\right\|_{L^{2}\left(\Sigma_{k j}\right)}^{2} .
$$

One can notice that the case of the identity operator corresponds to $s=0$ and $\Lambda_{j k}=\Lambda_{j k}^{*}=$ $I$. However, the interesting case, as said previously, will correspond to $s=\frac{1}{2}$.

Remark 6 In the sequel, it will be useful to set $T_{k k}=I$. We thus have $s=0$ for $T_{k k}$.

\subsubsection{Well-posedness of local problems}

We can now consider the model problems

$$
\left\{\begin{array}{l}
-\Delta u_{k}-\omega^{2} u_{k}=f_{k}, \quad \text { in } \Omega_{k} \\
\partial_{\nu_{k}} u_{k}+i \omega T_{j k}\left(u_{k / \Sigma_{k j}}\right)=g_{k j}, \quad \text { on } \Sigma_{k j} .
\end{array}\right.
$$

Here we must be precise on the meaning of a solution of (96) which implies to raise some subtle difficulties in the sense one gives to the boundary condition. If we look for a $u_{k}$ in $H^{1}\left(\Omega_{k}\right)$, then for $f \in L^{2}\left(\Omega_{k}\right)$, we shall also have $\Delta u_{k} \in L^{2}\left(\Omega_{k}\right)$ so that $\partial_{\nu_{k}} u_{k / \partial \Omega_{k}}$ belongs to $H^{-\frac{1}{2}}\left(\partial \Omega_{k}\right)$. It is known that the restriction of $\partial_{\nu_{k}} u_{k / \partial \Omega_{k}}$ to $\Sigma_{k j}$, namely $\partial_{\nu_{k}} u_{k / \Sigma_{k j}}$ can only be defined as an element of a space which strictly contains $H^{-\frac{1}{2}}\left(\partial \Omega_{k}\right)$, namely

$$
H_{00}^{\frac{1}{2}}\left(\Sigma_{k j}\right)^{\prime} \equiv \text { the dual space of } H_{00}^{\frac{1}{2}}\left(\Sigma_{k j}\right),
$$

where we remind that $H_{00}^{\frac{1}{2}}\left(\Sigma_{k j}\right)$ can be defined as the subspace of functions of $H^{\frac{1}{2}}\left(\Sigma_{k j}\right)$ whose extension by 0 belongs to $H^{\frac{1}{2}}\left(\partial \Omega_{k}\right.$ ) (see [24] or [22] for equivalent definitions and various properties of these spaces). Therefore, the equality

$$
\partial_{\nu_{k}} u_{k / \Sigma_{k j}}+i \omega T_{j k}\left(u_{k / \Sigma_{k j}}\right)=g_{k j},
$$

a priori holds in $H_{00}^{\frac{1}{2}}\left(\Sigma_{k j}\right)^{\prime}$. However, if $g_{k j}$ belongs to $H^{-\frac{1}{2}}\left(\Sigma_{k j}\right)$, we have, since $T_{j k} \in$ $\mathcal{L}\left(H^{s}\left(\Sigma_{k j}\right), H^{-s}\left(\Sigma_{k j}\right)\right)$ with $s \in\left[0, \frac{1}{2}\right]$

$$
\partial_{\nu_{k}} u_{k / \Sigma_{k j}} \in H^{-\frac{1}{2}}\left(\Sigma_{k j}\right) \text {. }
$$

In such a case, one has the following property

$$
\forall \varphi \in H^{\frac{1}{2}}\left(\partial \Omega_{k}\right) \quad<\partial_{\nu_{k}} u_{k}, \varphi>_{\partial \Omega_{k}}=\sum_{j}<\partial_{\nu_{k}} u_{k / \Sigma_{k j}}, \varphi / \Sigma_{k j}>_{\Sigma_{k j}},
$$


due to the density in $H^{\frac{1}{2}}\left(\partial \Omega_{k}\right)$ of the space of functions in $H^{\frac{1}{2}}\left(\partial \Omega_{k}\right)$ whose restriction to each $\Sigma_{k j}$ belongs to $H_{00}^{\frac{1}{2}}\left(\Sigma_{k j}\right)([24],[22])$. Therefore (54) is equivalent to the variational problem

Find $u_{k} \in H^{1}\left(\Omega_{k}\right)$ such that, $\forall v_{k} \in H^{1}\left(\Omega_{k}\right)$

$$
\begin{gathered}
\int_{\Omega_{k}}\left(\nabla u_{k} \nabla v_{k}-\omega^{2} u_{k} v_{k}\right) d x+i \omega \sum_{j}<T_{j k}\left(u_{k / \Sigma_{k j}}\right), \overline{v_{k}} / \Sigma_{k j}>_{\Sigma_{k j}} \\
=\int_{\Omega_{k}} f v_{k} d x+i \omega \sum_{j}<g_{k j}, \overline{v_{k}} / \Sigma_{k j}>_{\Sigma_{k j}} .
\end{gathered}
$$

\section{Theorem 3}

(i) Assume that $f \in L^{2}\left(\Omega_{k}\right)$ and $g_{k j} \in H^{-\frac{1}{2}}\left(\Sigma_{k j}\right)$ for any $j$, then (96) admits a unique solution $u_{k} \in H^{1}\left(\Omega_{k}\right)$.

(ii) If $g_{k j} \in H^{-s}\left(\Sigma_{k j}\right)$, for each $j$ than the quantities

$$
\left(\Lambda_{k j}^{*}\right)^{-1}\left(\partial_{\nu_{k}} u_{k / \Sigma_{k j}}\right) \pm i \omega \Lambda_{k j}\left(u_{k / \Sigma_{k j}}\right)
$$

belong to $L^{2}\left(\Sigma_{k j}\right)$ and one has the identity

$$
\begin{aligned}
& \sum_{j}\left\|\left(\Lambda_{k j}^{*}\right)^{-1}\left(\partial_{\nu_{k}} u_{k / \Sigma_{k j}}\right)+i \omega \Lambda_{k j}\left(u_{k / \Sigma_{k j}}\right)\right\|_{L^{2}\left(\Sigma_{k j}\right)}^{2} \\
= & \sum_{j}\left\|\left(\Lambda_{k j}^{*}\right)^{-1}\left(\partial_{\nu_{k}} u_{k / \Sigma_{k j}}\right)-i \omega \Lambda_{k j}\left(u_{k / \Sigma_{k j}}\right)\right\|_{L^{2}\left(\Sigma_{k j}\right)}^{2} .
\end{aligned}
$$

Proof : (i) It is easy to see that Fredholm's alternative applies to (100). We thus only have to check the uniqueness of the solution. We then consider $f=0$ and $g_{k j}=0$. Let us take $v_{k}=\overline{u_{k}}$ in (100). Taking the imaginary part of the resulting equality leads to

$$
\omega \sum_{j}\left\|\Lambda_{k j}\left(u_{k / \Sigma_{k j}}\right)\right\|_{L^{2}\left(\Sigma_{k j}\right)}^{2}=0 .
$$

Therefore $\Lambda_{k j}\left(u_{k / \Sigma_{k j}}\right)=0$ and by injectivity, $u_{k / \Sigma_{k j}}=0$. This is true for all $j$, therefore $u_{k / \partial \Omega_{k}}=0$. Going back to (100) with $f=g_{k j}=0$, we get

$$
\int_{\Omega_{k}}\left(\nabla u_{k} \nabla v_{k}-\omega^{2} u_{k} v_{k}\right) d x=0, \quad \forall v_{k} \in H^{1}\left(\Omega_{k}\right)
$$

which means that $\Delta u_{k}+\omega^{2} u_{k}=0$ and $\partial_{\nu_{k}} u_{k}=0$ on $\partial \Omega_{k}$. As we also have $u_{k / \partial \Omega_{k}}=0$ we conclude that $u_{k}=0$ by classical arguments ([11], page 211).

(ii) Note that

$$
\left(\Lambda_{k j}^{*}\right)^{-1}\left(\partial_{\nu_{k}} u_{k / \Sigma_{k j}}\right) \pm i \omega \Lambda_{k j}\left(u_{k / \Sigma_{k j}}\right)=\left(\Lambda_{k j}^{*}\right)^{-1}\left(\partial_{\nu_{k}} u_{k / \Sigma_{k j}} \pm i \omega T_{k j}\left(u_{k / \Sigma_{k j}}\right)\right) .
$$

As $\partial_{\nu_{k}} u_{k / \Sigma_{k j}}+i \omega T_{k j}\left(u_{k / \Sigma_{k j}}\right)=g_{k j}$ is $H^{-s}\left(\Sigma_{k j}\right)$ so is $\partial_{\nu_{k}} u_{k / \Sigma_{k j}}-i \omega T_{k j}\left(u_{k / \Sigma_{k j}}\right)=g_{k j}-$ $2 i \omega T_{k j}\left(u_{k / \Sigma_{k j}}\right)$. Furthermore, $\left(\Lambda_{k j}^{*}\right)^{-1} \in \mathcal{L}\left(H^{-s}\left(\Sigma_{k j}\right), L^{2}\left(\Sigma_{k j}\right)\right)$, this implies that the left 
hand side of (104) is in $L^{2}\left(\Sigma_{k j}\right)$. Moreover, we have

$$
\begin{gathered}
\left\|\left(\Lambda_{k j}^{*}\right)^{-1}\left(\partial_{\nu_{k}} u_{k / \Sigma_{k j}}\right) \pm i \omega \Lambda_{k j}\left(u_{k / \Sigma_{k j}}\right)\right\|_{L^{2}\left(\Sigma_{k j}\right)}^{2}=\left\|\left(\Lambda_{k j}^{*}\right)^{-1}\left(\partial_{\nu_{k}} u_{k / \Sigma_{k j}}\right)\right\|_{L^{2}\left(\Sigma_{k j}\right)}^{2} \\
\quad+\omega^{2}\left\|\Lambda_{k j}\left(u_{k / \Sigma_{k j}}\right)\right\|_{L^{2}\left(\Sigma_{k j}\right)}^{2} \pm 2 \omega \Im\left(\left(\Lambda_{k j}^{*}\right)^{-1}\left(\partial_{\nu_{k}} u_{k / \Sigma_{k j}}\right), \Lambda_{k j}\left(u_{k / \Sigma_{k j}}\right)\right)_{L^{2}\left(\Sigma_{k j}\right)} .
\end{gathered}
$$

To conclude, it suffices to prove that $\sum_{j}\left(\left(\Lambda_{k j}^{*}\right)^{-1}\left(\partial_{\nu_{k}} u_{k / \Sigma_{k j}}\right), \Lambda_{k j}\left(u_{k / \Sigma_{k j}}\right)\right)_{L^{2}\left(\Sigma_{k j}\right)}$ is real. At first, we remark that

$$
\left(\left(\Lambda_{k j}^{*}\right)^{-1} \partial_{\nu_{k}}\left(u_{k / \Sigma_{k j}}\right), \Lambda_{k j}\left(u_{k / \Sigma_{k j}}\right)\right)_{L^{2}\left(\Sigma_{k j}\right)}=<\partial_{\nu_{k}} u_{k}, u_{k}>_{\Sigma_{k j}}
$$

Thanks to (99) and Green's formula, we get

$$
\begin{aligned}
& \sum_{j}\left(\left(\Lambda_{k j}^{*}\right)^{-1}\left(\partial_{\nu_{k}} u_{k / \Sigma_{k j}}\right), \Lambda_{k j}\left(u_{k / \Sigma_{k j}}\right)\right)_{L^{2}\left(\Sigma_{k j}\right)}=<\partial_{\nu_{k}} u_{k}, u_{k}>_{\partial \Omega_{k}} \\
& =\int_{\Omega_{k}}\left(\left|\nabla u_{k}\right|^{2}-\omega^{2}\left|u_{k}\right|^{2}\right) d x=\text { some real quantity, }
\end{aligned}
$$

which concludes the proof.

\subsubsection{Reformulation at interfaces}

We generalize in this section the content of section 2.2. The new unknown at interfaces will be

$$
x=\left(x_{k}\right), \quad x_{k / \Sigma_{k j}}=-\left(\Lambda_{k j}^{*}\right)^{-1}\left(\partial_{\nu_{k}} u_{k / \Sigma_{k j}}\right)+i \omega \Lambda_{k j}\left(u_{k / \Sigma_{k j}}\right),
$$

where we have assumed that

$$
\partial_{\nu_{k}} u_{k / \Sigma_{k j}} \in H^{-s}\left(\Sigma_{k j}\right), \quad \forall k, j
$$

so that

$$
x \in V \text {. }
$$

We shall now give a new formulation at interfaces of the transmission problem (93). It will fit the same abstract framework than the one of section 2 modulo a new definition of operators $E, S$ and $A$.

Definition of $E, S$ and $A$ We define

$$
\begin{gathered}
E \in \mathcal{L}\left(V, \prod_{k=1}^{K} H^{1}\left(\Omega_{k}\right)\right), \\
e=\left(e_{k}\right) \in \prod_{k=1}^{K} H^{1}\left(\Omega_{k}\right)=E(y) \Leftrightarrow \\
\left\{\begin{array}{l}
-\Delta e_{k}-\omega^{2} e_{k}=0 \\
\left(\Lambda_{k j}^{*}\right)^{-1}\left(\partial_{\nu_{k}} e_{k / \Sigma_{k j}}\right)+i \omega \Lambda_{k j}\left(e_{k / \Sigma_{k j}}\right)=y_{k j} \text { on } \Sigma_{k j} .
\end{array}\right.
\end{gathered}
$$


The fact that the operator $E$ is well defined is a direct consequence of theorem 3 . By compactness of the embedding $H^{1}\left(\Omega_{k}\right) \hookrightarrow H^{\nu}\left(\Omega_{k}\right), \nu<1, E$ is compact from $V$ into $\prod_{k} H^{\nu}\left(\Omega_{k}\right)$. We have a more general result

Theorem $4 E$ is a compact operator from $V$ into $\prod_{k} H^{\nu}\left(\Omega_{k}\right)$ with $\nu<1$ in (94) if $s<\frac{1}{2}$, and $\nu=1$ if not.

Proof : We only need to look at the case $s<\frac{1}{2}$. Let $y^{n}$ a bounded sequence of $V$ and $e^{n}=E\left(y^{n}\right)$. Up to the extraction of a subsequence, there exits some $e$ such that

$$
\begin{array}{lll}
e^{n} \longrightarrow e & \text { in } \prod_{k} L^{2}\left(\Omega_{k}\right), & \text { strongly } \\
\nabla e^{n} \longrightarrow \nabla e & \text { in } \prod_{k} L^{2}\left(\Omega_{k}\right), & \text { weakly. }
\end{array}
$$

By trace theorem, $e_{/ \Sigma_{k j}}^{n}$ is bounded in $H^{\frac{1}{2}}\left(\partial \Sigma_{k j}\right)$. As $s<\frac{1}{2}$, we may assume

$$
e_{/ \Sigma_{k j}}^{n} \longrightarrow e_{/ \Sigma_{k j}} \text { in } H^{s}\left(\Sigma_{k j}\right) \text { strongly. }
$$

By Green's theorem, one has in each $\Omega_{k},\left(d_{k}^{n}=e_{k}^{n}-e, d_{k j}^{n}=d_{k / \Sigma_{k j}}^{n}\right)$

$$
\int_{\Omega_{k}}\left|\nabla d_{k}^{n}\right|^{2} d x=\omega^{2} \int_{\Omega_{k}}\left|d_{k}^{n}\right|^{2} d x+<\partial_{\nu_{k}} d_{k}^{n},\left(d_{k / \Omega_{k}}^{n}\right)>_{\partial \Omega_{k}}
$$

But we have

$$
\begin{gathered}
<\partial_{\nu_{k}} d_{k}^{n}, d_{k / \partial \Omega_{k}}^{n}>_{\partial \Omega_{k}}=\sum_{j}<\partial_{\nu_{k}} d_{k j}^{n}, d_{k j}^{n}>_{\Sigma_{k j}}=\sum_{j}\left(\left(\Lambda_{k j}^{*}\right)^{-1} \partial_{\nu_{k}} d_{k j}^{n}, \Lambda_{k j} d_{k j}^{n}\right)_{L^{2}\left(\Sigma_{k j}\right)} \\
=-i \omega \sum_{j}\left\|\Lambda_{k j} d_{k j}^{n}\right\|_{L^{2}\left(\Sigma_{k j}\right)}^{2}+\sum_{j}\left(\Lambda_{k j} d_{k j}^{n}, y_{k j}^{n}\right)_{L^{2}\left(\Sigma_{k j}\right)} .
\end{gathered}
$$

Property (114) implies $\Lambda_{k j} d_{k}^{n} \rightarrow 0$ in $L^{2}\left(\Sigma_{k j}\right)$ and therefore

$$
\left(\Lambda_{k j} d_{j k}^{n}, y_{k}^{n}\right)_{L^{2}\left(\Sigma_{k j}\right)} \longrightarrow 0
$$

we conclude

$$
\int_{\Omega_{k}}\left|\nabla\left(e_{k}^{n}-e_{k}\right)\right|^{2} d x=\int_{\Omega_{k}}\left|\nabla d_{k}^{n}\right|^{2} d x \quad \rightarrow \quad 0,
$$

which achieves the proof.

With the help of operator $E$ we can define $S \in \mathcal{L}(V, V)$ by

$$
x=S y \Leftrightarrow x_{k / \Sigma_{k j}}=x_{k j}=-\left(\Lambda_{k j}^{*}\right)^{-1}\left(\partial_{\nu_{k}} e_{k / \Sigma_{k j}}\right)+i \omega \Lambda_{k j}\left(e_{k / \Sigma_{k j}}\right), \quad e=E(y) .
$$

By theorem 3 -(ii), we know that $S$ is a unitary operator $S^{*} S=S S^{*}=I$. Next, we define $A \in \mathcal{L}(V, V)$

$$
A=S \Pi \text {. }
$$


Operator $A$ is clearly contractant and, following the proof of section 2 , one proves that $I-A$ is injective, thanks to the injectivity of $\Lambda_{k j}$.

In order to formulate the problem satisfied by $x_{k}$, we introduce $v=\left(v_{k}\right) \in \prod_{k} H^{1}\left(\Omega_{k}\right)$ where $v_{k}$ is solution of the local problem

$$
\left\{\begin{array}{l}
-\Delta v_{k}-\omega^{2} v_{k}=f_{k} \\
\left(\Lambda_{k j}^{*}\right)^{-1}\left(\partial_{\nu_{k}} v_{k / \Sigma_{k j}}\right)+i \omega \Lambda_{k j}\left(v_{k / \Sigma_{k j}}\right)=0 \text { on } \Sigma_{k j}
\end{array}\right.
$$

and let us set

$$
g_{k j}=-\left(\Lambda_{k j}^{*}\right)^{-1}\left(\partial_{\nu_{k}} v_{k / \Sigma_{k j}}\right)+i \omega \Lambda_{k j}\left(v_{k / \Sigma_{k j}}\right) .
$$

Following the proof of theorem 2, it is straightforward to establish the following result.

\section{Theorem 5}

(i) If $u=\left(u_{k}\right)$ is solution of (93), then $x \in V$ defined by (108) is solution of

$$
\mathcal{P}_{g}: \quad(I-A) x=g .
$$

(ii) Reciprocally, if $x$ is solution of $\left(\mathcal{P}_{g}\right)$, then $u=v+E(\Pi x)$ is solution of (93).

\subsubsection{The Domain Decomposition algorithms}

Once one has identified the spaces $V$ and the operators $E, S$ and $A$, it is easy to generalize what has been done in section 3.2.1. We define the relaxed Jacobi and Gauss Seidel Algorithms by replacing all the quantities $i \omega u_{k}$ by $i \omega T_{j k} u_{k}$ in (72) and (85). These algorithms corresponds to the iterative processes (76) and (87).

Finally and if we choose $\tilde{u}^{n}$ as the approximate value of the sought solution $u$, all our algorithms can be described in the general form

$$
\begin{aligned}
& \check{x}_{k j}^{0}=\Lambda_{k j}^{*} x_{k j}^{0} \text {, given in } H^{-s}\left(\Sigma_{k j}\right) \\
& \text { for } n=1, \ldots \\
& \text { for } k=1, \ldots, K \\
& \text { solve }\left\{\begin{array}{l}
\omega^{2} \tilde{u}_{k}^{n+1}+\Delta \tilde{u}_{k}^{n+1}=f, \text { in } \Omega_{k} \\
\partial_{\nu_{k}} \tilde{u}_{k}^{n+1}+i \omega T_{j k}\left(\tilde{u}_{k}^{n+1} / \Sigma_{k j}\right)=\check{x}_{j k}^{n^{\star}} \quad \text { on } \Sigma_{k j}, j \neq k \\
\partial_{\nu_{k}} \tilde{u}_{k}^{n+1}+i \omega \tilde{u}_{k}^{n+1}=0 \text { on } \Sigma_{k k} .
\end{array}\right. \\
& \text { update } \quad \check{x}_{k j}^{n+1}=(1-r) \check{x}_{k j}^{n}+r\left(-\partial_{\nu_{k}} \tilde{u}_{k}^{n+1}+i \omega T_{j k}\left(\tilde{u}_{k}^{n+1} / \Sigma_{k j}\right)\right) \text {, }
\end{aligned}
$$

with $\check{x}_{j k}^{n^{\star}}=\check{x}_{j k}^{n}$ except for the Gauss Seidel algorithm for which $\check{x}_{j k}^{n^{\star}}=\check{x}_{j k}^{n+1}$ when $j$ is less than $k$. This shows that it is not necessary to use the operator $\Lambda_{k j}$ but only $T_{j k}$. 


\section{Convergence analysis}

The aim of what follows is to establish some results of convergence of the two relaxed algorithms (Jacobi and Gauss Seidel) introduced in section 3. For the non-relaxed case, which is technically more complicated, we refer to [14]. We will work only with the abstract form of these algorithms, so that our proof is valid for both the first versions proposed in section 3.2 and the second one with the general interface operators considered in section 3.3 .

We begin with introducing some notations. We first define the error at the interface as the quantities

$$
\varepsilon^{n}=x^{n}-x \quad \in V,
$$

and the two errors committed on the unknown $u$ itself, depending whether one considers $u^{n}$ (formula (78)) or $\tilde{u}^{n}$ (formula (81)) for the approximation of $u$

$$
\begin{aligned}
& e^{n}=u^{n}-u \quad \in \prod_{k=1}^{K} H^{1}\left(\Omega_{k}\right) \\
& \tilde{e}^{n}=\tilde{u}^{n}-u \quad \in \prod_{k=1}^{K} H^{1}\left(\Omega_{k}\right) .
\end{aligned}
$$

The sequence $x^{n}$ satisfies the iterative process

$$
\varepsilon^{n+1}=(1-r) \varepsilon^{n}+r A \varepsilon^{n}
$$

in the case of the Jacobi's algorithm and

$$
\varepsilon^{n+1}=(1-r) \varepsilon^{n}+r\left(S \Pi^{+} \varepsilon^{n+1}+S \Pi^{-} \varepsilon^{n}\right),
$$

in the case of the Gauss-Seidel's algorithm. The two sequences $e^{n}$ and $\tilde{e}^{n}$ are then deduced from $\varepsilon^{n}$ by

$$
\begin{aligned}
& e^{n+1}=(1-r) E\left(S^{*} \varepsilon^{n}\right)+r E\left(\Pi \varepsilon^{n}\right) \\
& \tilde{e}^{n}=E\left(\Pi \varepsilon^{n}\right) .
\end{aligned}
$$

In the sequel, we are going to obtain two types of results :

- A convergence result for the general situation : we only obtain the convergence of $\varepsilon^{n}$ to 0 in the weak topology of $V$. However, thanks to the compactness properties of $E$ (cf Theorem 4) we shall obtain what we really want, namely the strong convergence of $u^{n}$ (or $\tilde{u}^{n}$ ) to $u$ at least in $L^{2}$. (We shall get even better - see section 4.2)

- A convergence result in a particular case for which we shall have strong convergence to 0 for $\varepsilon^{n}$. Moreover, this convergence will be exponentially fast and will imply the exponential decay of the errors $e^{n}$ (or $\tilde{e}^{n}$ ) in the $H^{1}$ topology of each sub-domain. We shall see in section 4.2 that our proof will require a particular geometrical property of the partition of $\Omega$ as well as an adequate choice for the operators $T_{j k}$. 


\subsection{A first general result}

The main tool of the analysis consists in a priori energy estimates that we deduce of two identities which are the object of the next lemma. It will be useful to introduce the following norm in $V(r \in] 0,1])$

$$
\|x\|_{r}^{2}=(1-r)\left\|P_{\Sigma}^{-} x\right\|^{2}+\left\|P_{\Gamma} x\right\|^{2}+\left\|P_{\Sigma}^{+} x\right\|^{2} .
$$

Note that this norm is equivalent to the usual norm

$$
(1-r)^{\frac{1}{2}}\|x\| \leq\|x\|_{r} \leq\|x\| .
$$

We also define the operator

$$
J_{r}=\left(I-r S \Pi^{-}\right)^{-1} \in \mathcal{L}(V) .
$$

(Note that $\left(I-r S \Pi^{-}\right)$is an isomorphism in $V$ : indeed, in the matrix representation of section 3 , it is block lower triangular, the triangular blocks being identity operators)

\section{Lemma 5}

(i) (Jacobi's algorithm) The sequence $\varepsilon^{n}$ satisfying (127) satisfies the identity

$$
\left\|\varepsilon^{n+1}\right\|^{2}=\left\|\varepsilon^{n}\right\|^{2}-r(1-r)\left\|(I-A) \varepsilon^{n}\right\|^{2}-r\left\|P_{\Gamma} \varepsilon^{n}\right\|^{2} .
$$

(ii) (Gauss-Seidel's algorithm) The sequence $\varepsilon^{n}$ satisfying (128) satisfies the identity

$$
\left\|\varepsilon^{n+1}\right\|_{r}^{2}=\left\|\varepsilon^{n}\right\|_{r}^{2}-r(1-r)\left\|J_{r}(I-A) \varepsilon^{n}\right\|^{2}-r\left\|P_{\Gamma} \varepsilon^{n}\right\|^{2} .
$$

Proof : (i) We consider the sequence $\varepsilon^{n}$ satisfying (127). We have, using $A=S \Pi$

$$
\left\|\varepsilon^{n+1}\right\|^{2}=(1-r)^{2}\left\|\varepsilon^{n}\right\|^{2}+r^{2}\left\|S \Pi \varepsilon^{n}\right\|^{2}+2 r(1-r) \Re\left(S \Pi \varepsilon^{n}, \varepsilon^{n}\right) .
$$

On the other hand, we use the identity

$$
\left\|(I-S \Pi) \varepsilon^{n}\right\|^{2}=\left\|\varepsilon^{n}\right\|^{2}+\left\|S \Pi \varepsilon^{n}\right\|^{2}-2 \Re\left(S \Pi \varepsilon^{n}, \varepsilon^{n}\right),
$$

to eliminate $\Re\left(S \Pi \varepsilon^{n}, \varepsilon^{n}\right)$ in (135). This leads to

$$
\left\|\varepsilon^{n+1}\right\|^{2}=(1-r)\left\|\varepsilon^{n}\right\|^{2}+r\left\|S \Pi \varepsilon^{n}\right\|^{2}-2 r(1-r)\left\|(I-A) \varepsilon^{n}\right\|^{2} .
$$

Using lemma 2 and the fact that $S$ is an isometry, we get

$$
\left\|S \Pi \varepsilon^{n}\right\|^{2}=\left\|\Pi \varepsilon^{n}\right\|^{2}=\left\|\varepsilon^{n}\right\|^{2}-\left\|P_{\Gamma} \varepsilon^{n}\right\|^{2} .
$$

Plugging (137) in (135) then leads to (133). 
(ii) Let us now consider a sequence $\varepsilon^{n}$ satisfying (127). We have

$$
\begin{aligned}
\left\|\varepsilon^{n+1}\right\|^{2}= & (1-r)^{2}\left\|\varepsilon^{n}\right\|^{2}+r^{2}\left\|S\left(\Pi^{+} \varepsilon^{n}+\Pi^{-} \varepsilon^{n+1}\right)\right\|^{2} \\
& +2 r(1-r) \Re\left(\varepsilon^{n}, S\left(\Pi^{+} \varepsilon^{n}+\Pi^{-} \varepsilon^{n+1}\right)\right) .
\end{aligned}
$$

Applying the identity $2 \Re(x, S y)=\|x\|^{2}+\|y\|^{2}-\|x-S y\|^{2}$, to $x=\varepsilon^{n}$ and $y=\Pi^{+} \varepsilon^{n}+$ $\Pi^{-} \varepsilon^{n+1}$, we get, using also the fact that $S$ is an isometry

$$
\begin{aligned}
\left\|\varepsilon^{n+1}\right\|^{2}= & (1-r)^{2}\left\|\varepsilon^{n}\right\|^{2}+r^{2}\left\|\Pi^{+} \varepsilon^{n}+\Pi^{-} \varepsilon^{n+1}\right\|^{2} \\
& +r(1-r)\left(\left\|\varepsilon^{n}\right\|^{2}+\left\|\Pi^{+} \varepsilon^{n}+\Pi^{-} \varepsilon^{n+1}\right\|^{2}\right) \\
& -r(1-r)\left\|\varepsilon^{n}-S\left(\Pi^{+} \varepsilon^{n}+\Pi^{-} \varepsilon^{n+1}\right)\right\|^{2}
\end{aligned}
$$

or, equivalently

$$
\begin{aligned}
\left\|\varepsilon^{n+1}\right\|^{2}= & (1-r)\left\|\varepsilon^{n}\right\|^{2}+r\left\|\Pi^{+} \varepsilon^{n}+\Pi^{-} \varepsilon^{n+1}\right\|^{2} \\
& -r(1-r)\left\|\varepsilon^{n}-S\left(\Pi^{+} \varepsilon^{n}+\Pi^{-} \varepsilon^{n+1}\right)\right\|^{2} .
\end{aligned}
$$

Since, by lemma 3 ,

$$
\left\|\Pi^{+} x+\Pi^{-} y\right\|^{2}=\left\|\Pi^{+} x\right\|^{2}+\left\|\Pi^{-} y\right\|^{2}=\left\|P_{\Sigma}^{+} x\right\|^{2}+\left\|P_{\Sigma}^{-} y\right\|^{2},
$$

we obtain, introducing $z^{n}=\varepsilon^{n}-S\left(\Pi^{+} \varepsilon^{n}+\Pi^{-} \varepsilon^{n+1}\right)$

$$
\left\|\varepsilon^{n+1}\right\|^{2}=(1-r)\left\|\varepsilon^{n}\right\|^{2}+r\left\|P_{\Sigma}^{+} \varepsilon^{n}\right\|^{2}+r\left\|P_{\Sigma}^{-} \varepsilon^{n+1}\right\|^{2}-r(1-r)\left\|z^{n}\right\|^{2} .
$$

We then notice that, by definition of the norm $\|\cdot\|_{r}$

$$
\begin{aligned}
& \left\|\varepsilon^{n+1}\right\|^{2}-r\left\|P_{\Sigma}^{-} \varepsilon^{n+1}\right\|^{2}=\left\|\varepsilon^{n+1}\right\|_{r}^{2} \\
& (1-r)\left\|\varepsilon^{n}\right\|^{2}+r\left\|P_{\Sigma}^{+} \varepsilon^{n}\right\|^{2}=\left\|\varepsilon^{n}\right\|_{r}^{2}-r\left\|P_{\Gamma} \varepsilon^{n}\right\|^{2},
\end{aligned}
$$

so that (142) can be rewritten

$$
\left\|\varepsilon^{n+1}\right\|_{r}^{2}=\left\|\varepsilon^{n}\right\|_{r}^{2}-r(1-r)\left\|z^{n}\right\|^{2}-r\left\|P_{\Gamma} \varepsilon^{n}\right\|^{2},
$$

which is nothing but (134) if we show that $z^{n}=J_{r}(I-A) \varepsilon^{n}$. But from (128) we deduce

$$
\varepsilon^{n+1}=\varepsilon^{n}-r z^{n} .
$$

Equation (128) also shows that $\varepsilon^{n+1}$ is related to $\varepsilon^{n}$ by

$$
\left(I-r S \Pi^{-}\right) \varepsilon^{n+1}=(1-r) x^{n}+r S \Pi^{+} \varepsilon^{n} .
$$

Therefore, multiplying (145) by $\left(I-r S \Pi^{-}\right)$we get

$$
(1-r) x^{n}+r S \Pi^{+} \varepsilon^{n}=\varepsilon^{n}-r S \Pi^{-} \varepsilon^{n}-r\left(I-r S \Pi^{-}\right) z^{n},
$$

from which

$$
\left(I-r S \Pi^{-}\right) z^{n}=(I-A) \varepsilon^{n}
$$

what we wanted to show.

A direct consequence of this lemma is 
Theorem 6 Assume that $0<r<1$, the sequence $\varepsilon^{n}$ given by (127) (or (128)) converges weakly to 0 in the space $V$ and we have

$$
\begin{aligned}
& u^{n} \longrightarrow u \quad \text { strongly in } \prod_{k=1}^{K} H^{\nu}\left(\Omega_{k}\right) \\
& \tilde{u}^{n} \longrightarrow u \quad \text { strongly in } \prod_{k=1}^{K} H^{\nu}\left(\Omega_{k}\right),
\end{aligned}
$$

where $\nu=1$ if $s<\frac{1}{2}$ in (94) for all $T_{j k}$ and $\nu<1$ if not.

Proof : We only give the proof for the Jacobi's algorithm using (133). The proof for the Gauss-Seidel's algorithm via (134) is almost identical thanks to the facts that $\|\cdot\|_{r}$ is a norm on $V$ equivalent to $\|$.$\| . Identity (133) implies in particular that$

$$
\begin{aligned}
& \varepsilon^{n} \text { is bounded in } V \quad\left(\left\|\varepsilon^{n}\right\| \text { decreases }\right) \\
& \sum_{n=0}^{+\infty}\left\|(I-A) \varepsilon^{n}\right\|^{2}<+\infty
\end{aligned}
$$

Therefore, up to the extraction of a subsequence still denoted by $\varepsilon^{n}$, we know that there exists $\varepsilon \in V$ such that

$$
\varepsilon^{n} \longrightarrow \varepsilon \quad \text { weakly in } . V
$$

Moreover, from (150) we also now that

$$
(I-A) \varepsilon^{n} \longrightarrow 0 \quad \text { strongly in } V .
$$

Therefore, by linearity of $A$, we get

$$
(I-A) \varepsilon=0
$$

which yields $\varepsilon=0$ since $I-A$ is injective. Note also that, as 0 is the only possible limit, the whole sequence converges.

The end of the proof is nothing but a straightforward consequence of the compactness properties of the operator $E$ (given in theorem 4) and of the two equalities (129).

Remark 7 A careful examination of our derivation of the a priori energy estimates shows that the equalities (133) and (134) becomes inequalities if we replace the hypothesis $\|S x\|=$ $\|x\|$ by $\|S x\| \leq\|x\|$ (S contractant instead of isometric). We could use this remark to extend our result to damped Helmholtz equations as considered in [18] for example.

\subsection{A case of exponential convergence}

In this section, we shall assume that the partition $\left(\Omega_{k}\right)_{k}$ of $\Omega$ has the following geometrical property.

For each $(j, k), \partial \Omega_{k} \cap \partial \Omega_{j}$ is either empty either

a closed manifold of dimension $N-1$. 
The second hypothesis turns on the value of $s$ for the operators $T_{j k}, j \neq k\left(s=\frac{1}{2}\right)$

$$
\begin{gathered}
T_{j k}=\Lambda_{j k}^{*} \Lambda_{j k}, \text { with } \Lambda_{j k} \text { isomorphism from } \\
H^{\frac{1}{2}}\left(\partial \Omega_{k} \cap \partial \Omega_{j}\right) \text { into } L^{2}\left(\partial \Omega_{k} \cap \partial \Omega_{j}\right) .
\end{gathered}
$$

In such a situation, we have the following fundamental result

Lemma 6 Under both assumptions (154) and (155) $I-A$ is one to one in $V$.

Proof : As $I-A$ is always injective, we only have to prove that it is onto. Let $g$ be given in $V$, we look for an $x$ such that

$$
(I-A) x=g .
$$

If such an $x$ exists, then $u=\left(u_{k}\right)=E(x)$ satisfies in each $\Omega_{k}$

$$
\left\{\begin{array}{l}
u_{k} \in H^{1}\left(\Omega_{k}\right), \quad-\Delta u_{k}-\omega^{2} u_{k}=0, \quad \text { in } \Omega_{k} \\
\left(\Lambda_{k j}^{*}\right)^{-1}\left(\partial_{\nu_{k}} u_{k / \Sigma_{k j}}\right)+i \omega \Lambda_{k j}\left(u_{k / \Sigma_{k j}}\right) \\
=-\left(\Lambda_{k j}^{*}\right)^{-1}\left(\partial_{\nu_{j}} u_{j / \Sigma_{j k}}\right)+i \omega \Lambda_{k j}\left(u_{j / \Sigma_{j k}}\right)+g_{k j} \quad \text { on } \Sigma_{k j}, k \neq j \\
\partial_{\nu_{k}} u_{k}+i \omega u_{k}=g_{k k} \text { on } \Sigma_{k k},
\end{array}\right.
$$

which is equivalent to

$$
\left\{\begin{array}{l}
u_{k} \in H^{1}\left(\Omega_{k}\right), \quad-\Delta u_{k}-\omega^{2} u_{k}=0, \quad \text { in } \Omega_{k} \\
\partial_{\nu_{k}} u_{k}+\partial_{\nu_{j}} u_{j}=\Lambda_{k j}^{*} \frac{g_{k j}+g_{j k}}{2}, \quad \text { on } \Sigma_{k j}, k<j \\
\left(u_{k}-u_{j}\right)=\left(\Lambda_{k j}\right)^{-1} \frac{g_{k j}-g_{j k}}{2 i \omega}, \quad \text { on } \Sigma_{k j}, k<j \\
\partial_{\nu_{k}} u_{k}+i \omega u_{k}=g_{k k} \text { on } \Sigma_{k k} .
\end{array}\right.
$$

Reciprocally, if there exists an $u \in H^{1}\left(\Omega_{k}\right)$ solution of (158), one constructs a solution of $(I-A) x=g$ via

$$
x=\left(x_{k}\right), \quad x_{k / \Sigma_{k j}}=-\left(\Lambda_{k j}^{*}\right)^{-1}\left(\partial_{\nu_{k}} u_{k / \Sigma_{k j}}\right)+i \omega \Lambda_{k j}\left(u_{k / \Sigma_{k j}}\right) .
$$

Let us define

$$
\varphi_{k j}=-\varphi_{j k}=\left(\Lambda_{k j}\right)^{-1} \frac{g_{k j}-g_{j k}}{2 i \omega} \in H^{\frac{1}{2}}\left(\Sigma_{k j}\right),
$$

and let us assume for a while that one can find a $u^{*} \in \prod_{k} H^{1}\left(\Omega_{k}\right)$ with $\Delta u_{k}^{*} \in L^{2}\left(\Omega_{k}\right)$,

$$
u_{k / \Sigma_{k j}}^{*}-u_{j / \Sigma_{j k}}^{*}=\varphi_{k j}, \quad \partial_{\nu_{k}} u_{k / \Sigma_{k j}}^{*}+\partial_{\nu_{j}} u_{j / \Sigma_{j k}}^{*} \in H^{-\frac{1}{2}}\left(\Sigma_{k j}\right), k \neq j,
$$

then, setting

$$
u=u^{*}+w
$$


function $w$ must satisfy

$$
\left\{\begin{array}{l}
w \in H^{1}(\Omega), \quad-\Delta w_{k}-\omega^{2} w_{k}=\Delta u_{k}^{*}+\omega^{2} u_{k}^{*}, \quad \text { in } \Omega_{k} \\
\partial_{\nu_{k}} w_{k}+\partial_{\nu_{j}} w_{j}=\tilde{g}_{k j} \quad \text { on } \Sigma_{k j}, k \neq j \\
\partial_{\nu_{k}} w_{k}+i \omega w_{k}=\tilde{g}_{k k} \quad \text { on } \Sigma_{k k},
\end{array}\right.
$$

with

$$
\begin{aligned}
& \tilde{g}_{k k}=g_{k k}-\partial_{\nu_{k}} u_{k / \Sigma_{k k}}^{*}-i \omega u_{k / \Sigma_{k k}}^{*} \\
& \tilde{g}_{k j}=\Lambda_{k j}^{*} \frac{g_{k j}+g_{j k}}{2}-\partial_{\nu_{k}} u_{k / \Sigma_{k j}}^{*}-\partial_{\nu_{j}} u_{j / \Sigma_{j k}}^{*},
\end{aligned}
$$

The variational formulation of this problem is

$$
\text { Find } w \in H^{1}(\Omega) \text { such that, } \forall v \in H^{1}(\Omega)
$$

$$
\begin{aligned}
& \int_{\Omega}\left(\nabla w \nabla v-\omega^{2} w v\right) d x+i \omega \int_{\Gamma} w v d \sigma \\
& =\sum \int_{\Omega_{k}}\left(\Delta u_{k}^{*}+\omega^{2} u_{k}^{*}\right) v_{k} d x+\sum_{k} \sum_{j}<\tilde{g}_{k j}, \bar{v}_{/ \Sigma_{k j}}>_{\Sigma_{k j}} .
\end{aligned}
$$

Problem $(165)$ is a nice variational problem in $H^{1}(\Omega)$ since the $\tilde{g}_{k j} \in H^{-\frac{1}{2}}\left(\Sigma_{k j}\right)$. The existence of $w$ is easily obtained by Fredholm's alternative. The problem is thus reduced to the construction of $u^{*}$. For $k \leq j$, we define $u_{k j}$ as the solutions in $H^{1}\left(\Omega_{k}\right)$ of the following elliptic problem

$$
\begin{cases}-\Delta u_{k j}+u_{k j}=0, & \text { in } \Omega_{k} \\ u_{k j / \Sigma_{k j}}=\tilde{\varphi}_{k j}, & \text { on } \partial \Omega_{k}\end{cases}
$$

with

$$
\tilde{\varphi}_{k j}=\varphi_{k j} \text { on } \Sigma_{k j}, \quad \tilde{\varphi}_{k j}=0 \text { on } \Sigma_{k \ell}, \ell \neq j .
$$

As all the manifolds $\Sigma_{k j}$ are assumed to be closed, all the $\tilde{\varphi}_{k j}$ are functions of $H^{\frac{1}{2}}\left(\Omega_{k}\right)$ and the existence of $u_{k j}$ is simply obtained using Lax-Milgram theory and the trace theorem. Moreover, as $\Sigma_{k j}$ is closed

$$
\partial_{\nu_{k}} u_{k j / \Sigma_{k j}} \in H^{-\frac{1}{2}}\left(\Sigma_{k j}\right), \quad \partial_{\nu_{k}} u_{k j / \Sigma_{k k}} \in H^{-\frac{1}{2}}\left(\Sigma_{k k}\right) .
$$

Then it is not difficult to check that a good candidate for $u^{*}$ is

$$
u^{*}=\sum_{k<j} u_{k j}
$$

and the proof of lemma 6 is complete. 
Remark 8 The theory of potential, [10], gives us a more explicit formulae for $(I-A)^{-1} g$ (however restricted to the case of constant coefficients). Indeed, one can verify that

$$
\left.x_{k j}=-\left(\Lambda_{k j}^{*}\right)^{-1}\left(\partial_{\nu_{k}}\left(e_{k}+e_{0}\right)_{/ \Sigma_{k j}}\right)+i \omega \Lambda_{k j}\left(e_{k}+e_{0}\right)_{/ \Sigma_{k j}}\right) \text {, }
$$

where

$$
\begin{aligned}
e(x)=\sum_{k} & \sum_{j<k}\left[\int_{\Sigma_{k j}} \Phi(x, y) \frac{1}{2} \Lambda_{j k}^{*}\left(g_{k j}+g_{j k}\right)(y) d \sigma(y)\right. \\
& \left.-\int_{\Sigma_{k j}} \partial_{\nu(y)} \Phi(x, y) \frac{1}{2 \omega} \Lambda_{j k}^{-1}\left(g_{k j}-g_{j k}\right)(y) d \sigma(y)\right],
\end{aligned}
$$

where $\Phi(x, y)$ is a Green's function for the Helmholtz equation and $e_{0}$ is the solution of

$$
\left\{\begin{array}{l}
-\omega^{2} e_{0}-\Delta e_{0}=0, \quad \text { in } \Omega \\
\partial_{\nu} e_{0}+i \omega e_{0}=g_{k k}-\partial_{\nu} e-i \omega e, \quad \text { on } \Gamma .
\end{array}\right.
$$

As consequence of lemma 6

Theorem 7 (Exponential convergence) Under both assumptions (154) and (155), for each value $r \in] 0,1[$, there exists a real number $\tau \in] 0,1[$ such that

$$
\left\|\varepsilon^{n}\right\|_{V} \leq C \tau^{n}
$$

Consequently, we also have

$$
\left\|u-u^{n}\right\|_{\Pi_{k}\left(H^{1}\left(\Omega_{k}\right)\right)} \leq C \tau^{n}, \quad\left\|u-\tilde{u}^{n}\right\|_{\Pi_{k}\left(H^{1}\left(\Omega_{k}\right)\right)} \leq C \tau^{n} .
$$

Proof : We only give the proof in the case of Jacobi's algorithm. The proof for GaussSeidel is almost the same. Since $(I-A)$ is an isomorphism in $V$, there exists a $\delta>0$ such that

$$
\|(I-A)\| \geq \delta\|x\|,
$$

(note that $\delta \leq 1$, since $I-A$ is contractant). Therefore from identity (133), we get

$$
\left\|\varepsilon^{n+1}\right\|^{2} \leq\left(1-r(1-r) \delta^{2}\right)\left\|\varepsilon^{n}\right\|^{2}
$$

which gives (173) with $C=\left\|\varepsilon_{0}\right\|$ and $\tau=\sqrt{1-r(1-r) \delta^{2}}$. The rest of the proof is a consequence of the continuity of $E$.

Remark 9 In our proof, the best (i.e. the smallest) value of $\tau$ is given by $r=\frac{1}{2}$ which maximizes $r(1-r)$. We shall come back on this point in section 5 .

In order to be complete, it remains to give examples of operators $T_{j k}$ satisfying (155). The most natural one consists in considering fractional powers of the Laplace-Beltrami operator. More precisely, assuming that $\Sigma_{k j}$ is a sufficiently regular manifold, we denote 
by $\Delta_{j k}$ the Laplace-Beltrami operator on $\Sigma_{k j}$ ([32], tome 1, page 137-139), i.e. the selfadjoint operator in $L^{2}\left(\Sigma_{k j}\right)$ associated to the bilinear form with domain $H^{1}\left(\Sigma_{k j}\right)$ :

$$
a_{j k}(\varphi, \psi)=\int_{\Sigma_{k j}} \nabla_{\Gamma} \varphi \cdot \nabla_{\Gamma} \psi d \sigma
$$

where $\nabla_{\Gamma}$ denotes the usual tangential gradient operator on $\Sigma_{k j}$. It is then well known that if $\alpha$ and $\beta$ are two positive real numbers, one can define the positive selfadjoint operator in $L^{2}\left(\Sigma_{k j}\right)$ with domain $H^{1}\left(\Sigma_{k j}\right)$

$$
T_{j k}=\left(\alpha I-\frac{\beta}{\omega^{2}} \Delta_{j k}\right)^{\frac{1}{2}}
$$

It is well known that $T_{j k}$ can be extended in a unique way as an isomorphism from $H^{\frac{1}{2}}\left(\Sigma_{k j}\right)$ onto $H^{-\frac{1}{2}}\left(\Sigma_{k j}\right)$ as soon as $\alpha$ and $\beta$ are strictly positive. One then satisfies (155) with

$$
\Lambda_{j k}=\left(\alpha I-\frac{\beta}{\omega^{2}} \Delta_{j k}\right)^{\frac{1}{4}}
$$

As an example, let us consider the $2 \mathrm{D}$ case where $\Sigma_{k j}$ is the circle of radius $a$, that we can parametrize by the polar angle $\theta$. In such a case the operator $T_{j k}$ given by (178) is diagonalized in the orthonormal basis of $L^{2}\left(\Sigma_{k j}\right)$ defined by

$$
e_{n}(\theta)=(2 \pi a)^{\frac{1}{2}} e^{i n \theta} \quad n \in Z
$$

and we have

$$
T_{j k} e_{n}=\left(\alpha+\beta \frac{n^{2}}{\omega^{2} a^{2}}\right) e_{n} .
$$

One can note that the operator defined by (178) is non local (it is a convolution-type operator) and will produce full matrices after space discretization (cf section 6). A possible compromise with purely local would consist in considering an integral operator of the form ( $\delta$ denoting some strictly positive parameter)

$$
\begin{aligned}
< & T_{j k} \varphi, \psi>=\alpha \int_{\Sigma_{j k}} \varphi(x) \bar{\psi}(x) d \sigma(x) \\
& +\beta \int_{\Sigma_{k j}} \int_{\Sigma_{k j}} G(x-y) \chi_{\delta}(|x-y|)(\operatorname{curl} \varphi \wedge \nu)(y)(\operatorname{curl} \bar{\psi} \wedge \nu)(x) d \sigma(x) d \sigma(y)
\end{aligned}
$$

where $G$ is for instance the fundamental solution of the Laplace operator in $R^{N}$

$$
G(x)=\frac{1}{2 \pi} \log |x| \quad N=2 \quad G(x)=\frac{1}{4 \pi|x|} \quad N=3,
$$

and $\chi_{\delta}(r), r>0$ a smooth positive cut-off function satisfying :

$$
\begin{array}{ll}
\chi_{\delta}(r)=1 & \text { if } r<\delta \\
\chi_{\delta}(r)=0 & \text { if } r>2 \delta,
\end{array}
$$


whose role is to "localize" the operator $T_{j k}$ (the "local" character of $T_{j k}$ then depending on the value of $\delta$ ) Such a choice has not yet be tested up to our knowledge. Our conjecture is that such an operator possesses the good properties ensuring exponential convergence : the important fact is that the kernel $G$ has the correct singularity for $x=0$.

Remark 10 The choice of the operator $T_{j k}$ defined by (178) ( $\Delta_{j k}$ being associated to (177) in $H^{1}\left(\Sigma_{k j}\right)$ ) can also be made even in the case where $\Sigma_{k j}$ is not a closed manifold (the Laplace-Beltrami operator then corresponds to a "Neumann condition" on $\left.\partial \Sigma_{k j}\right)$. Although our proof of exponential convergence is no longer valid, numerical experiments ([20]) show that this improves the convergence by comparison with the case $T_{j k}=I$.

\section{Determination of the rate of convergence in a simple case}

\subsection{Presentation of the example, notations.}

To get more insight in the rates of convergence of the method, we propose to analyze a very simple $2 \mathrm{D}$ example. We consider the square $\Omega=]-\frac{L}{2}, \frac{L}{2}[\times] 0, L[$ decomposed into two rectangles similar in size $\Omega_{1}$ and $\Omega_{2}$, with only one interface (cf figure 2). The DDM is applied to the solution of the Helmholtz equation in $\Omega$ with our usual conditions $\partial_{\nu}+i \omega u=$ 0 at left and right sides and a Neumann condition at top and bottom sides.

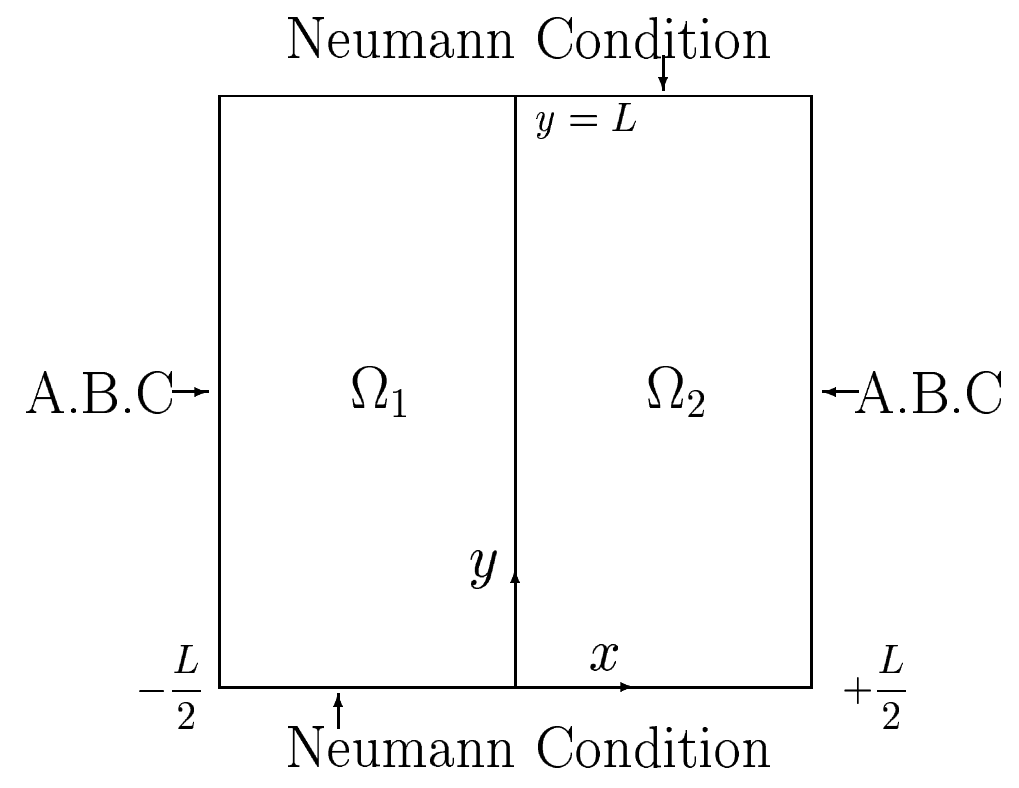

Figure 2: Geometry of the simple model 
We shall used in this section the presentation of the algorithm we gave in subsection 3.3.4. Hence, we construct two sequences $\tilde{u}_{1}^{n}$ and $\tilde{u}_{2}^{n}$ which approximate $\left(u_{1}, u_{2}\right)$, restrictions to $\Omega_{1}$ and $\Omega_{2}$ of $u$. The equations for $\tilde{u}_{1}^{n}$ and $\tilde{u}_{2}^{n}$ are

$$
\begin{gathered}
\left\{\begin{array}{l}
-\omega^{2} \tilde{u}_{k}^{n}-\Delta \tilde{u}_{k}^{n}=f_{k}, \quad k=1,2 \\
\partial_{y} \tilde{u}_{k}^{n}(x, 0)=\partial_{y} \tilde{u}_{k}^{n}(x, L)=0, \quad k=1,2
\end{array}\right. \\
\left\{\begin{array}{l}
\left(-\partial_{x} \tilde{u}_{1}^{n}+i \omega \tilde{u}_{1}^{n}\right)\left(-\frac{L}{2}, y\right)=0 \\
\left(\partial_{x} \tilde{u}_{2}^{n}+i \omega \tilde{u}_{2}^{n}\right)\left(+\frac{L}{2}, y\right)=0 .
\end{array}\right.
\end{gathered}
$$

The coupling between two successive iterations $n$ and $n+1$ is made at the level of the interface $x=0$

$$
\begin{aligned}
& \left(\Lambda^{*}\right)^{-1} \partial_{x} \tilde{u}_{1}^{n+1} / x=0+i \omega \Lambda \tilde{u}_{1}^{n+1} / x=0=x_{21}^{n} \\
& -\left(\Lambda^{*}\right)^{-1} \partial_{x} \tilde{u}_{2}^{n+1} / x=0+i \omega \Lambda \tilde{u}_{2}^{n+1} / x=0=x_{12}^{n^{\star}},
\end{aligned}
$$

where $n^{\star}=n$ (Jacobi) or $n^{\star}=n+1$ (Gauss Seidel). The operator $\Lambda$ is chosen among a two positive parameters family $\left(\Lambda\right.$ is a bijection from $H^{\frac{1}{2}}(0, L)$ onto $L^{2}(0, L)$ as soon as $\beta>0$.)

$$
\Lambda=\Lambda_{\alpha, \beta}=\left(\alpha I d-\frac{\beta}{\omega^{2}} \partial_{y y}^{2}\right)^{\frac{1}{4}} .
$$

For a given relaxation parameter $r$, the $x_{12}^{n}$ and $x_{21}^{n}$ are updated by the formula

$$
\begin{aligned}
& x_{12}^{n+1}=(1-r) x_{12}^{n}+r\left(-\left(\Lambda^{*}\right)^{-1} \partial_{x} \tilde{u}_{1}^{n+1} / x=0+i \omega \Lambda \tilde{u}_{1}^{n+1} / x=0\right) \\
& x_{21}^{n+1}=(1-r) x_{21}^{n}+r\left(\left(\Lambda^{*}\right)^{-1} \partial_{x} \tilde{u}_{2}^{n+1} / x=0+i \omega \Lambda \tilde{u}_{2}^{n+1} / x=0\right) .
\end{aligned}
$$

From the general theory, we already know that the error $\tilde{e}_{k}^{n}=\tilde{u}_{k}^{n}-u_{k}, k=1,2$ converges to zero with the index of iteration $n$. The aim of what follows is to determine the rate of this convergence. At first, the errors satisfy to both the Helmholtz equation with zero second term and the absorbing boundary condition at $x=+\frac{L}{2}(k=2)$ and $x=-\frac{L}{2}(k=1)$. Using Theorem 3, we deduce the estimates

$$
\begin{aligned}
\left\|\tilde{e}_{1}^{n+1}\right\|_{H^{1}\left(\Omega_{1}\right)}^{2} & \leq C_{0} \|\left(\Lambda^{*}\right)^{-1} \partial_{x} \tilde{e}_{1}^{n+1} / x=0+i \omega \Lambda \tilde{e}_{1}^{n+1} / x=0 \\
& \leq\left\|_{L^{2}(0, L)}^{2}\right\| \varepsilon_{21}^{n} \|_{L^{2}(0, L)}^{2} \\
\left\|\tilde{e}_{2}^{n+1}\right\|_{H^{1}\left(\Omega_{2}\right)}^{2} & \leq C_{0} \|\left(-\left(\Lambda^{*}\right)^{-1} \partial_{x} \tilde{e}_{2}^{n+1} / x=0\right. \\
& \leq C_{0}\left\|\varepsilon_{12}^{n^{\star}}\right\|_{L^{2}(0, L)}^{2} .
\end{aligned}
$$

where $\varepsilon_{12}^{n}$ and $\varepsilon_{21}^{n}$ are the errors at interfaces. They satisfy to

$$
\begin{aligned}
& \varepsilon_{12}^{n+1}=(1-r) \varepsilon_{12}^{n}+r\left(-\left(\Lambda^{*}\right)^{-1} \partial_{x} \tilde{e}_{1}^{n+1} / x=0+i \omega \Lambda \tilde{e}_{1}^{n+1} / x=0\right) \\
& \varepsilon_{12}^{n}=(1-r) \varepsilon_{12}^{n}-r\left(\left(\Lambda^{*}\right)^{-1} \partial_{x} \tilde{e}_{2}^{n+1} / x=0+i \omega \Lambda \tilde{e}_{2}^{n+1} / x=0\right) .
\end{aligned}
$$


In the next subsection, we derive explicit expressions for the errors $\varepsilon_{12}^{n}$ and $\varepsilon_{21}^{n}$ which lead us to sharp error estimates.

\subsection{A general error estimate}

The solution of this sequence of problems can be explicitely derived with the help of a technique of separation of variables. We introduce,

$$
\begin{aligned}
& \tilde{e}_{k}^{n}(x, y)=\sum_{p=0}^{\infty} \hat{e}_{k, p}^{n}(x) w_{p}(y), k=1,2 \\
& \varepsilon_{k j}^{n}=\sum_{p=0}^{\infty} \hat{\varepsilon}_{k j, p}^{n} w_{p}(y), k j=12 \text { or } 21,
\end{aligned}
$$

where functions $w_{p}(y)$ are the eigenvectors of $\partial_{y y}$ with Neumann boundary conditions and are also eigenfunctions of $\Lambda_{\alpha, \beta}$

$$
\left\{\begin{array}{l}
w_{p}(y)=\sqrt{\frac{2}{L}} \cos \left(\frac{\pi}{L}\left(y-\frac{L}{2}\right)\right), \\
\Lambda_{\alpha, \beta} w_{p}=\sqrt{t_{p}^{\alpha \cdot \beta}} w_{p}, \quad t_{p}^{\alpha \cdot \beta}=\left(\alpha+\frac{\beta \pi^{2}}{\omega^{2} L^{2}} p^{2}\right)^{\frac{1}{2}} \\
\left(\omega^{2}+\Delta\right) w_{p}=k_{p}^{2} w_{p}+\partial_{x x}^{2} w_{p} \quad k_{p}=\sqrt{\omega^{2}-\frac{\pi^{2} p^{2}}{L^{2}}}
\end{array}\right.
$$

(the precise choice of the sign for the complex square root defining $k_{p}$ does not really matter).

The quantities $\hat{e}_{k, p}^{n}(x)$ are Fourier coefficients of the error. We shall say that $\hat{e}_{k, p}^{n}(x)$ is the mode of order $p$ of the error term $\tilde{e}_{k}^{n}$. Each of them satisfies a reduced homogeneous Helmholtz equation

$$
k_{p}^{2} \hat{e}_{k, p}^{n+1}+\partial_{x x}^{2} \hat{e}_{k, p}^{n+1}=0
$$

in their respective domains. These equations are solved according to

$$
\begin{aligned}
& \hat{e}_{1, p}^{n+1}=a_{1, p}^{n+1} \exp \left(i k_{p} x\right)+b_{1, p}^{n+1} \exp \left(-i k_{p} x\right) \\
& \hat{e}_{2, p}^{n+1}=a_{2, p}^{n+1} \exp \left(-i k_{p} x\right)+b_{2, p}^{n+1} \exp \left(i k_{p} x\right) .
\end{aligned}
$$

Boundary conditions at $x=0$ and $x= \pm \frac{L}{2}$ allows us to determine $a_{k, p}^{n+1}$ and $b_{k, p}^{n+1}$ as a function of the $\hat{\varepsilon}_{12, p}^{n}$ and $\hat{\varepsilon}_{21, p}^{n}$.

$$
A_{p}\left[\begin{array}{l}
a_{1, p}^{n+1} \\
b_{1, p}^{n+1}
\end{array}\right]=\hat{\varepsilon}_{21, p}^{n}\left[\begin{array}{c}
\sqrt{t_{p}^{\alpha, \beta}} \\
0
\end{array}\right], \quad A_{p}\left[\begin{array}{c}
a_{2, p}^{n+1} \\
b_{2, p}^{n+1}
\end{array}\right]=\hat{\varepsilon}_{12, p}^{n^{\star}}\left[\begin{array}{c}
\sqrt{t_{p}^{\alpha, \beta}} \\
0
\end{array}\right],
$$

with

$$
A_{p}=\left[\begin{array}{ccc}
i k_{p}+i \omega t_{p}^{\alpha, \beta} & -i k_{p}+i \omega t_{p}^{\alpha, \beta} \\
\left(-i k_{p}+i \omega\right) e^{-i k_{p} L / 2} & , & \left(i k_{p}+i \omega\right) e^{+i k_{p} L / 2}
\end{array}\right]
$$


Our goal is to obtain a recursive relation for $\left(\hat{\varepsilon}_{12, p}^{n}, \hat{\varepsilon}_{21, p}^{n}\right)$. Equation (191) implies the following equality for the Fourier components of the error at interface

$$
\begin{aligned}
& \hat{\varepsilon}_{12, p}^{n+1}=(1-r) \hat{\varepsilon}_{12, p}^{n}+r\left(-\left(t_{p}^{\alpha, \beta}\right)^{-\frac{1}{2}} \partial_{x} \hat{e}_{1, p}^{n+1} / x=0+i \omega\left(t_{p}^{\alpha, \beta}\right)^{\frac{1}{2}} \hat{e}_{1, p}^{n+1} / x=0\right) \\
& \hat{\varepsilon}_{21, p}^{n+1}=(1-r) \hat{\varepsilon}_{21, p}^{n}+r\left(\left(t_{p}^{\alpha, \beta}\right)^{-\frac{1}{2}} \partial_{x} \hat{e}_{2, p}^{n+1} / x=0+i \omega\left(t_{p}^{\alpha, \beta}\right)^{\frac{1}{2}} \hat{e}_{2, p}^{n+1} / x=0\right) .
\end{aligned}
$$

or, using (195)

$$
\begin{aligned}
& \hat{\varepsilon}_{12, p}^{n+1}=(1-r) \hat{\varepsilon}_{12, p}^{n}+\frac{r}{\sqrt{t^{\alpha, \beta}}}\left(\left(i k_{p}+i \omega t_{p}^{\alpha, \beta}\right) a_{1, p}^{n+1}+\left(-i k_{p}+i \omega t_{p}^{\alpha, \beta}\right) b_{1, p}^{n+1}\right) \\
& \hat{\varepsilon}_{21, p}^{n+1}=(1-r) \hat{\varepsilon}_{21, p}^{n}+\frac{r}{\sqrt{t^{\alpha, \beta}}}\left(\left(k_{p}+\omega t_{p}^{\alpha, \beta}\right) a_{2, p}^{n+1}+\left(-k_{p}+\omega t_{p}^{\alpha, \beta}\right) b_{2, p}^{n+1}\right) .
\end{aligned}
$$

Via (196), we can eliminate the $a_{k, p}^{n+1}$ and $b_{k, p}^{n+1}$ and obtain a relationship between two successive iterates. We obtain

$$
\begin{aligned}
& \hat{\varepsilon}_{12, p}^{n+1}=(1-r) \hat{\varepsilon}_{12, p}^{n}+r \lambda_{p} \hat{\varepsilon}_{21, p}^{n} \\
& \hat{\varepsilon}_{21, p}^{n+1}=(1-r) \hat{\varepsilon}_{21, p}^{n}+r \lambda_{p} \hat{\varepsilon}_{12, p}^{n^{\star}}
\end{aligned}
$$

with

$$
\lambda_{p}=\frac{\left(-k_{p}+\omega t_{p}^{\alpha, \beta}\right)\left(k_{p}+\omega\right) e^{i \frac{k_{p} L}{2}}-\left(k_{p}+\omega t_{p}^{\alpha, \beta}\right)\left(-k_{p}+\omega\right) e^{-i \frac{k_{p} L}{2}}}{\left(k_{p}+\omega t_{p}^{\alpha, \beta}\right)\left(k_{p}+\omega\right) e^{i \frac{k_{p} L}{2}}-\left(-k_{p}+\omega t_{p}^{\alpha, \beta}\right)\left(-k_{p}+\omega\right) e^{-i \frac{k_{p} L}{2}}} .
$$

Equation (200) can be written in the matrix form

$$
\left[\begin{array}{c}
\hat{\varepsilon}_{12, p}^{n+1} \\
\hat{\varepsilon}_{21, p}^{n+1}
\end{array}\right]=M_{p}(r)\left[\begin{array}{c}
\hat{\varepsilon}_{12, p}^{n} \\
\hat{\varepsilon}_{21, p}^{n}
\end{array}\right]
$$

with

$$
\text { Jacobi: } \quad M_{p}(r)=\left[\begin{array}{ccc}
1-r & , & r \lambda_{p} \\
r \lambda_{p} & , & (1-r)
\end{array}\right]
$$$$
\text { Gauss Seidel: } \quad M_{p}(r)=\left[\begin{array}{cc}
1-r & r \lambda_{p} \\
r(1-r) \lambda_{p} & (1-r)+r^{2} \lambda_{p}^{2}
\end{array}\right] .
$$

From (202), we deduce the sharp estimate (with Jacobi at least, the equality can occur)

$$
\left|\hat{\varepsilon}_{12, p}^{n+1}\right|^{2}+\left|\hat{\varepsilon}_{21, p}^{n+1}\right|^{2} \leq\left\|M_{p}(r)\right\|_{2}^{2 n}\left(\left|\hat{\varepsilon}_{12, p}^{0}\right|^{2}+\left|\hat{\varepsilon}_{21, p}^{0}\right|^{2}\right)
$$

where $\|M\|_{2}$ denotes the maximum of the square root of the eigenvalues of the matrix $M^{*} M$. Of course, the general theory of convergence indicates us that :

$$
\forall p, \quad\left\|M_{p}(r)\right\|_{2}<1
$$


This can also be checked by a direct computation for the Jacobi's algorithm using $\left|\lambda_{p}\right|<1$ (see the forthcoming section 5.3) but is less obvious for the Gauss-Seidel algorithm. Now, we define

$$
\tau_{r}=\sup _{p}\left\|M_{p}(r)\right\|_{2},
$$

and using Plancherel-Parceval's theorem

$$
\left\|\varepsilon_{j k}^{n}\right\|_{L^{2}(0, L)}^{2}=\sum_{p}\left|\hat{\varepsilon}_{j k, p}^{n}\right|^{2}
$$

inequality (205) leads to the estimate give us immediatly

$$
\left\|\tilde{e}_{1}^{n+1}\right\|_{H^{1}\left(\Omega_{1}\right)} \leq C \tau_{r}^{n} \quad,\left\|\tilde{e}_{2}^{n+1}\right\|_{H^{1}\left(\Omega_{1}\right)} \leq C \tau_{r}^{n^{\star}}
$$

with

$$
C^{2}=C_{0}^{2}\left\|\varepsilon_{12}^{0}\right\|_{L^{2}(0, L)}^{2}+C_{0}^{2}\left\|\varepsilon_{21}^{0}\right\|_{L^{2}(0, L)}^{2} .
$$

As a consequence, a geometrical convergence occurs when $\tau_{r}$ is strictly less than 1 . Moreover, $\tau_{r}$ governs the rate of convergence : it is thus the right quantity to analyse.

\subsection{Analysis of the non zero eigenvalues of $A$}

It is not difficult to realize that the $\lambda_{p}$ 's are nothing else than the non zero eigenvalues of operator $A\left(A x=0\right.$, if $x$ is localized on the boundaries $\left.\Sigma_{k k}\right)$. As $A$ is a contraction, all the $\left|\lambda_{p}\right|$ must be less than 1 . We distinguish different cases according to $k_{p}$ is real, purely imaginary or zero

- If $\frac{p \pi}{\omega L}<1$ : The mode is propagative, $k_{p}$ is real, equal to $\omega \sqrt{1-\frac{p^{2} \pi^{2}}{\omega^{2} L^{2}}}$ and

$$
\lambda_{p}=\frac{\cos \left(\frac{k_{p} L}{2}\right) k_{p} \omega\left(t_{p}^{\alpha, \beta}-1\right)+i \sin \left(\frac{k_{p} L}{2}\right)\left(\omega^{2} t_{p}^{\alpha, \beta}-k_{p}^{2}\right)}{\cos \left(\frac{k_{p} L}{2}\right) k_{p} \omega\left(t_{p}^{\alpha, \beta}+1\right)+i \sin \left(\frac{k_{p} L}{2}\right)\left(\omega^{2} t_{p}^{\alpha, \beta}+k_{p}^{2}\right)} .
$$

- If $\frac{p \pi}{\omega L}>1$ : The mode is evanescent, $k_{p}$ is imaginary, equal to $i \xi_{p}=i \sqrt{\frac{p^{2} \pi^{2}}{\omega^{2} L^{2}}-1}$ and

$$
\lambda_{p}=\frac{i \cosh \left(\frac{\xi_{p} L}{2}\right) \xi_{p} \omega\left(t_{p}^{\alpha, \beta}-1\right)-\sinh \left(\frac{\xi_{p} L}{2}\right)\left(\omega^{2} t_{p}^{\alpha, \beta}+\xi_{p}^{2}\right)}{i \cosh \left(\frac{\xi_{p} L}{2}\right) \xi_{p} \omega\left(t_{p}^{\alpha, \beta}+1\right)-\sinh \left(\frac{\xi_{p} L}{2}\right)\left(\omega^{2} t_{p}^{\alpha, \beta}-\xi_{p}^{2}\right)} .
$$

- If $\frac{p \pi}{\omega L}=1: k_{p}=0$ and

$$
\lambda_{p}=\lambda^{*} \equiv \frac{\sqrt{\alpha+\beta}-1+i \frac{\omega L}{2} \sqrt{\alpha+\beta}}{\sqrt{\alpha+\beta}+1+i \frac{\omega L}{2} \sqrt{\alpha+\beta}}
$$

In each case, it is not difficult to check that the modulus of $\lambda_{p}$ is always strictly less than 1 as we might expect. Analyzing what happens respectively for small and large values of $p$ permits us to distinguish the role of the parameters $\alpha$ and $\beta$ in the convergence process. Indeed : 
- When $p$ is 0 , we get

$$
\lambda_{0}=\frac{\sqrt{\alpha}-1}{\sqrt{\alpha}+1} .
$$

which shows that $\alpha$ will govern essentially the convergence of the low frequencies.

- When $p$ grows to infinity, we get

$$
\lim _{p \rightarrow \infty} \lambda_{p} \equiv \lambda_{\infty}=\frac{i \sqrt{\beta}-1}{i \sqrt{\beta}+1}
$$

which shows that $\beta$ will govern essentially the convergence of the high frequencies.

In particular (215) shows that

$$
\lim _{p \rightarrow \infty}\left|\lambda_{p}\right|=1
$$

what implies that $\|A\|$ is equal to 1 : this demonstrates, in this particular case, that the formal series $\sum A^{n}$ does not converge absolutely.

\subsection{The convergence rate for the Jacobi's algorithm}

Let us first check that for the Jacobi's algorithm, one gets exponential convergence as soon as $\beta \neq 0$ and $r \in] 0,1[$. One computes easily that

$$
\left\|M_{p}(r)\right\|_{2}=\max _{ \pm}\left|1-r \pm r \lambda_{p}\right|<1 \text {. }
$$

The only delicate point is to ensure that the $\lambda_{p}$ do not accumulate near \pm 1 in which case $\left\|M_{p}(r)\right\|_{2}$ would tend to 1 for all values of $r$ and $\tau_{r}$ would be 1 . According to (215), we have

$$
\tau_{r}^{\infty}=\lim _{p \rightarrow+\infty}\left\|M_{p}(r)\right\|_{2}=\max _{ \pm}\left|1-r \pm r \lambda_{\infty}\right|,
$$

which is strictly smaller than 1 for $\beta \neq 0$ and $r \in] 0,1[$ (see figure).

Remark 11 Here is the point where we can recover the precise role played by operator $T=\Lambda^{*} \Lambda$. The fact that $T$ is a pseudo-differential operator of first order reads in the behavior of its symbol $t_{p}^{\alpha, \beta}$ when $p$ becomes large: $t_{p}^{\alpha, \beta} \sim C p$. Note that this behavior is the only possibility to get the limit in (215) not equal to \pm 1 .

Remark 12 When $\beta=0$ (local operator), it is easy to see that $\tau_{r}^{\infty}=1$ which implies $\tau_{r}=1$. In this case, the convergence is not exponential and the estimate (210) is not sufficient to prove the convergence. One then has to take into account how the quantity $\left(\left|\hat{\varepsilon}_{12, p}^{0}\right|^{2}+\left|\hat{\varepsilon}_{21, p}^{0}\right|^{2}\right)$ occuring in (205) goes to zero when $p$ goes to infinity, which involves the regularity on the interface of the exact solution. In [14], it is shown that, if the solution is smooth one gets, for any $\varepsilon>0$

$$
\left\|\tilde{e}_{1}^{n}\right\|_{H^{1}\left(\Omega_{1}\right)}+\left\|\tilde{e}_{2}^{n}\right\|_{H^{1}\left(\Omega_{2}\right)} \leq \frac{C_{\varepsilon}}{n^{1-\varepsilon}}
$$


for $r \neq 1 / 2$ and

$$
\left\|\tilde{e}_{1}^{n}\right\|_{H^{1}\left(\Omega_{1}\right)}+\left\|\tilde{e}_{2}^{n}\right\|_{H^{1}\left(\Omega_{2}\right)} \leq \frac{C_{\varepsilon}}{n^{3 / 2-\varepsilon}}
$$

for $r=1 / 2$.

Of course the next step of the analysis consist is answering the question : what is the choice for the parameters $r, \alpha$ and $\beta$ which will minimize $\left|\tau_{r}\right|$ ? As we are going to see, a first naive analysis based on the examination of low and high frequencies would lead to choose $\alpha=1, \beta=1$ and $r=1 / 2$. However, if one looks at a typical curve $p \longrightarrow\left\|M_{p}(r)\right\|_{2}$ one sees that one has to distinguish three regions for $p$.

- High frequencies : $\frac{p \pi}{\omega L}>>1$

For these values, the convergence is essentially governed by

$$
\tau_{r}^{\infty}=\max _{ \pm}\left|1-r \pm r \lambda_{\infty}\right|
$$

It is then straigthforward to realize that the best way to damp these evanescent modes is to pick $\beta=1$ and $r=\frac{1}{2}$ : the $\lambda_{p}$ 's accumulates near $i$ and $\left\|M_{p}\left(r=\frac{1}{2}\right)\right\|_{2}$ tends to $\frac{1}{\sqrt{2}}$.

- Low frequencies : $\frac{p \pi}{\omega L}<<1$

For these values, the convergence is essentially governed by

$$
\tau_{r}^{0}=\max _{ \pm}\left|1-r \pm r \lambda_{0}\right|
$$

Using (214), one sees that the best value for $\alpha$ is 1 .

Note that if one stops the discussion here, the size $L$ of the subdomains has no influence on our choice.

- Resonant frequencies : $\frac{p \pi}{\omega L} \simeq 1$

These values of $p$ correspond to a local maximum of the curve $p \longrightarrow\left\|M_{p}(r)\right\|_{2}$, which is also the global maximum in a lot of cases. The convergence of the related modes is now governed by

$$
\tau_{r}^{*}=\max _{ \pm}\left|1-r \pm r \lambda^{*}\right|,
$$

where $\lambda^{*}$ is given by (213), which shows that trying to minimize $\tau_{r}^{*}$ involves the value of $\omega L$. In particular, $\lambda^{*}$ tends to 1 when $\omega L$ tends to $+\infty$, whatever the value of $(\alpha, \beta)$ or $r$ is. 


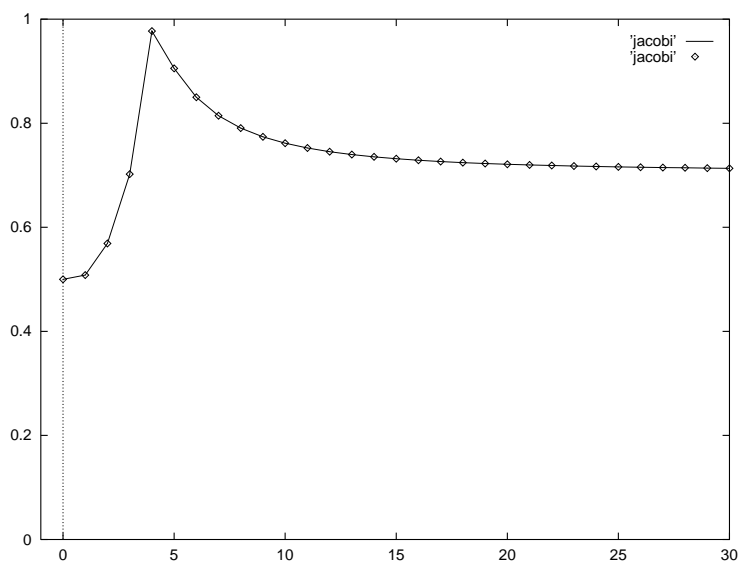

Figure 3: Domain of two wavelenghts: $\tau_{p}$ in function of $p$ for $r=\frac{1}{2}, \alpha=\beta=1$

It appears then that a good strategy for choosing $\alpha, \beta$ and $r$ consists in minimizing, $\omega L$ being given, the quantity

$$
\max \left\{\tau_{r}^{0}, \tau_{r}^{*}, \tau_{r}^{\infty}\right\}
$$

which can easily be done, at least numerically. We present the results of this optimization procedure in Figure 4 where, for different values of the frequency (from $\omega L=\pi$ which corresponds to $\mathrm{L}=0.5$ wavelengthes to $\omega L=10 \pi$ which corresponds to $\mathrm{L}=5$ wavelengthes), we plot the curves $p \longrightarrow\left\|M_{p}(r)\right\|_{2}$ for the optimal values of $(\alpha, \beta, r)$ which are given in Table 1, as well as the optimal value of the convergence rate. We observe experimentally that the optimal value of $(\alpha, \beta, r)$ is the one which ensures the equality

$$
\tau_{r}^{0}=\tau_{r}^{*}=\tau_{r}^{\infty}
$$

Also, we notice that the optimal rate decreases when the frequency increases which is not surprising and at least coherent with the fact $\tau^{*}$ tends to 1 when $\omega L$ tends to $+\infty$. Note also that for large frequencies $\alpha_{o p t}$ and $\beta_{o p t}$ are really different from 1 while $r_{o p t}$ remains closed to $\frac{1}{2}$.

\begin{tabular}{|c|c|c|c|c|}
\hline & $\omega L=\pi$ & $\omega L=2 \pi$ & $\omega L=4 \pi$ & $\omega L=10 \pi$ \\
\hline$\alpha$ & 0.11982 & 0.0269 & 0.007435 & 0.000384 \\
\hline$\beta$ & 0.85500 & 0.55 & 0.23076 & 0.0032 \\
\hline$r$ & 0.5160 & 0.645 & 0.5160 & 0.54126 \\
\hline$\tau_{r}$ & 0.734588 & 0.821 & 0.903 & 0.9792 \\
\hline
\end{tabular}

Table 1: Optimal parameters for 4 differents lenght of domain (Jacobi)

\subsection{The convergence rate for the Gauss Seidel's algorithm}

We do not reproduce the discussion which is essentially the same than for the Jacobi's algorithm except the fact that the computations are heavier. We only present in Figure 5 



Figure 4: Optimal curves $p \rightarrow\left\|M_{p}(r)\right\|^{2}$ for the Jacobi's algorithm $(\omega L=\pi$ :top left, $\omega L=2 \pi$ :top right, $\omega L=4 \pi$ :bottom left, $\omega L=10 \pi$ : bottom right.)

\begin{tabular}{|c|c|c|c|c|}
\hline & $\omega L=\pi$ & $\omega L=2 \pi$ & $\omega L=4 \pi$ & $\omega L=10 \pi$ \\
\hline$\alpha$ & 0.1538 & 0.0512 & 0.00641 & 0.001948 \\
\hline$\beta$ & 0.87179 & 0.43589 & 0.0871 & 0.0317 \\
\hline$r$ & 0.5 & 0.55128 & 0.4151 & 0.3235 \\
\hline$\tau_{r}$ & 0.5029 & 0.604 & 0.8921 & 0.9654 \\
\hline
\end{tabular}

Table 2: Optimal parameters for 4 differents lengthes of domain(Gauss Seidel)

and in Table 2 the results of the research optimal parameters. Let us emphasize the fact that, for a given value of $\omega L$, we get a better convergence with the Gauss-Seidel algorithm than with the Jacobi's algorithm.

\section{Other applications}

One of the interests of having identified a general framework for our domain decomposition method is to simplify the presentation of the extension of this type of method to other physical model with a complete guaranty of convergence : the abstract convergence proof remains true once one has verified that the operators $E, S$ and $A$ have the good proper- 



Figure 5: Optimal curves $p \rightarrow\left\|M_{p}(r)\right\|^{2}$ for the Gauss Seidel's algorithm $(\omega L=\pi$ :top left, $\omega L=2 \pi$ :top right, $\omega L=4 \pi$ :bottom left, $\omega L=10 \pi$ : bottom right.)

ties. In section 6.2, we shall consider the two important examples of the elastodynamics equations and of the 3D Maxwell's system.

Another advantage of our abstract framework is to include the discrete problems obtained after approximation in space, provided a good choice of the approximation process. We are going to show this in section 6.1 .

\subsection{Space discretization and domain decomposition}

In this section, we go back to our model problem $\left(P_{\Omega}^{f}\right)$ in dimension $N=2$ or 3 , and consider the case where the operator $\Lambda_{j k}$ is defined by (cf section 4 )

$$
\Lambda_{j k}=\left(\alpha I-\frac{\beta}{\omega^{2}} \Delta_{j k}\right)^{\frac{1}{4}}
$$

and assume for simplicity that the $\Omega_{k}$ 's are polyhedral domains. The importance of this section lies in the fact the convergence of the algorithm for the continuous problem does not imply the convergence for any discrete approximation. Initial numerical experiments performed by B. Despres (personal communication) show that a naïve finite-difference approximation of the interface conditions might lead to unstable iterative algorithms.

In order to avoid this kind of accident, the first major idea is that the discretization should have the same variational structure than the continuous. This leads naturally to 
consider finite element methods. In this class of methods, the choice of mixed-hybrid finite element methods was guided by the fact that the quantities that one wants to transmit at interfaces, namely the trace of the solution and of its normal derivative, were easily and consistently computable from the degrees of freedom, which is not the case for instance with Lagrange elements. Finally, we realized that this choice permitted to put the resulting discrete problem within our general framework of sections 2 and 3 , ensuring the convergence of the iterative method. This is of course the point of view we are going to adopt in our presentation. In the rest of this section, we assume for simplicity that the $\Omega_{k}$ 's are polyhedral domains. We restrict ourselves to the lower order element for simplicity.

The meshes For each sub-domain $\Omega_{k}$, we introduce a finite element triangulation $\mathcal{T}_{h}\left(\Omega_{k}\right)$ (of triangles in 2D, tetrahedra in 3D) of step-size $h>0$. We shall denote by $K$ the current element of $\mathcal{T}_{h}\left(\Omega_{k}\right)$. Moreover, for each interface $\Sigma_{j k}$, we shall assume that the traces of the two triangulations $\mathcal{T}_{h}\left(\Omega_{k}\right)$ and $\mathcal{T}_{h}\left(\Omega_{j}\right)$ coïncide with one single surface triangulation of $\Sigma_{j k}$, denoted. $\mathcal{T}_{h}\left(\Sigma_{j k}\right)$ : in other words, we work with matching grids. We shall denote by $T$ the current element of such a discretization. We also denote by $\mathcal{T}_{h}\left(\Sigma_{k k}\right)$ the trace of $\mathcal{T}_{h}\left(\Omega_{k}\right)$ on $\Sigma_{k k}$.

The space $V^{h}$ and the unknown $x^{h} \quad$ Here, we identify the space $V^{h}$ which will play the role of the space $V$ of section 2 . We have

$$
V^{h}=\prod_{j, k=1}^{K} V_{k j}^{h} \subset V,
$$

where

$$
V_{k j}^{h}=\left\{x_{k j}^{h} \in L^{2}\left(\Sigma_{j k}\right) / \forall T \in \mathcal{T}_{h}\left(\Sigma_{j k}\right), x_{k j / T}^{h} \in P_{0}(T)\right\} .
$$

Of course we shall look for $x^{h} \in V^{h}$ as an approximation of $x \in V$. Let us notice that

$$
\Pi V^{h} \subset V^{h}
$$

defined by (26).

The operators $E^{h}, S^{h}$ and $A^{h}$ Let us admit for a while the existence of selfadjoint operators

$$
\Lambda_{k j}^{h} \in \mathcal{L}\left(V_{k j}^{h}\right)
$$

which is supposed to be an approximation of $\Lambda_{k j}$. In order to define $E^{h}$, we first introduce a space $M^{h}$ for the approximation of $\prod_{k=1}^{K} L^{2}\left(\Omega_{k}\right)$

$$
M^{h}=\prod_{k=1}^{K} M_{k}^{h},
$$

where

$$
M_{k}^{h}=\left\{u_{k}^{h} \in L^{2}\left(\Omega_{k}\right) / \forall K \in \mathcal{T}_{h}\left(\Omega_{k}\right), u_{k / K}^{h} \in P_{0}(K)\right\},
$$


and a space $X^{h}$ for the approximation of $\prod_{k=1}^{K} H\left(\operatorname{div} ; \Omega_{k}\right)$

$$
X^{h}=\prod_{k=1}^{K} X_{k}^{h}
$$

where

$$
X_{k}^{h}=\left\{p_{k}^{h} \in H\left(\operatorname{div} ; \Omega_{k}\right) / \forall K \in \mathcal{T}_{h}\left(\Omega_{k}\right), p_{k / K}^{h} \in R T_{0}(K)\right\},
$$

where $R T_{0}(K)$ denotes the usual Raviart-Thomas-Nedelec space of lowest order, [29]. Then, we can define

$$
E^{h} \in \mathcal{L}\left(V^{h}, M^{h}\right)
$$

such that

$$
e^{h}=\left(e_{k}^{h}\right)=E^{h}\left(y^{h}\right),
$$

if and only if $\left(e^{h}, p^{h}, t^{h}\right) \in M^{h} \times X^{h} \times V^{h}$ is the solution of the following collection of local variational problems

$$
\begin{array}{lc}
\int_{\Omega_{k}} p_{k}^{h} \cdot q_{k}^{h} d x+\int_{\Omega_{k}} e_{k}^{h} \operatorname{div} q_{k}^{h} d x=\sum_{j} \int_{\Sigma_{k j}} t_{k j}^{h} q_{k}^{h} \cdot \nu_{k} d \sigma & \forall q_{k}^{h} \in X_{k}^{h} \\
-\int_{\Omega_{k}} \operatorname{div} p_{k}^{h} \cdot v_{k}^{h} d x-\omega^{2} \int_{\Omega_{k}} e_{k}^{h} v_{k}^{h} d x=\int_{\Omega_{k}} f v_{k}^{h} d x & \forall v_{k}^{h} \in M_{k}^{h} \\
\left(\Lambda_{k j}^{h}\right)^{-1}\left(p_{k}^{h} \cdot \nu_{k / \Sigma_{k j}}\right)+i \omega \Lambda_{k j}^{h}\left(e_{k / \Sigma_{k j}}^{h}\right)=y_{k j}^{h} & \text { in } V_{k j}^{h},
\end{array}
$$

which is nothing but a standard mixed-hybrid approximation of the local problem defining $e_{k}$ in (31). We then define

$$
S^{h} \in \mathcal{L}\left(V^{h}\right),
$$

by expressing that

$$
x^{h}=S^{h} y^{h}
$$

if and only if

$$
\begin{gathered}
\forall(k, j), \quad x_{k j}^{h}=-\left(\Lambda_{k j}^{h}\right)^{-1}\left(p_{k}^{h} \cdot \nu_{k / \Sigma_{k j}}\right)+i \omega \Lambda_{k j}^{h} e_{k / \Sigma_{k j}}^{h}, \\
\text { with }\left(e_{k}^{h}, p_{k}^{h}, t_{k}^{h}\right) \text { solution of }(231) .
\end{gathered}
$$

The reader will easily check that $S^{h}$ is an isometry, that $A^{h}$ is a contraction and that $I-A^{h}$ is invertible. For completeness we need to explain how one can construct $\Lambda_{j k}^{h}$. The idea is to define

$$
\Lambda_{j k}^{h}=\left(\alpha I-\frac{\beta}{\omega^{2}} \Delta_{j k}^{h}\right)^{\frac{1}{4}}
$$

where $\Delta_{j k}^{h} \in \mathcal{L}\left(V_{j k}^{h}\right)$ is an approximation of the Laplace-Beltrami operator $\Delta_{j k}$, that one can construct using mixed finite elements as follows. We first define the space

$$
\Phi_{j k}^{h}=\left\{\varphi_{j k}^{h} \in H\left(\operatorname{div}_{\Gamma} ; \Sigma_{j k}\right) / \forall T \in \mathcal{T}^{h}\left(\Sigma_{j k}\right), \varphi_{j k}^{h} \in R T_{0}(T)\right\}
$$


and we define

$$
y_{j k}^{h}=-\Delta_{j k}^{h} x_{j k}^{h}
$$

if and only if

$$
\begin{array}{ll}
\int_{\Sigma_{j k}} y_{j k}^{h} z_{j k}^{h} d \sigma=\int_{\Sigma_{j k}} \operatorname{div} \varphi_{j k}^{h} z_{j k}^{h} d \sigma & \forall z_{j k}^{h} \in V_{j k}^{h} \\
\int_{\Sigma_{j k}} \varphi_{j k}^{h} \cdot \psi_{j k}^{h} d \sigma=-\int_{\Sigma_{j k}} x_{j k}^{h} \operatorname{div} \psi_{j k}^{h} d \sigma & \forall \psi_{j k}^{h} \in \Phi_{j k}^{h} .
\end{array}
$$

Note that in practice, one has to construct the matrix representing $-\Delta_{j k}^{h}$ and to diagonalize this matrix in order to compute $\Lambda_{j k}^{h}$.

\subsection{Other physical models}

We present here how to adapt our domain decomposition method to problems whose unknown are vector fields. We try essentially to keep the notation of the previous section and only mention the new ones when we feel this is useful. For simplicity, we only present the case where the operators $\Lambda_{j k}$ are identity operators. For brevity, we simply make precise what is the interface unknown $x$, the space $V$ and the operators $E, S$ and $A$ which permits us to apply our abstract iterative methods. We let the reader check that the required properties are satisfied. This is straightforward in the case of elastodynamics equations, but less obvious for Maxwell's equations (we give some indications in this case).

We do not speak at all here of the discretization of these problems. The extension of the content of section 6.1 is easy provided that one uses appropriate mixed elements for elasticity ([29],[4]). The case of Maxwell's equations is less standard and requires the hybridization of the mixed elements of Nedelec [28]. We refer the reader to [17] or [9].

\subsubsection{Elastodynamics equations}

Notation In what follows

- $\Omega \subset R^{N}$ is the (bounded) propagation medium

- $\rho$ is the density of this medium

- $u=\left(u_{i}\right)$ denotes the displacement field

- $\varepsilon(u)=\left(\left(\varepsilon_{i j}(u)\right)\right)$ is the strain tensor

- $\left.\sigma_{(} u\right)=\left(\left(\sigma_{i j}(u)\right)\right)$ is the stress tensor

Let us recall that, for small deformations

$$
\varepsilon_{i j}(u)=\frac{1}{2}\left(\frac{\partial u_{i}}{\partial x_{j}}+\frac{\partial u_{j}}{\partial x_{i}}\right),
$$

and that in a linear medium, $\sigma(u)$ and $\varepsilon(u)$ are related by the Hooke's law

$$
\sigma(u)=C \cdot \varepsilon(u)
$$

where $C$ is a fourth-order tensor characteristic of the medium. 
The model problem For a given source term $f \in L^{2}(\Omega)^{N}$, we consider the problem

$$
\left\{\begin{array}{lr}
\text { Find } u \in H^{1}(\Omega)^{N} \text { such that } & \\
-\operatorname{div} \sigma(u)-\rho \omega^{2} u=f & \text { in } \Omega \\
\sigma(u) \nu+i \omega \rho u=0 & \text { on } \partial \Omega .
\end{array}\right.
$$

The space $V$ and the unknown $x \quad$ In this case we have

$$
\begin{aligned}
& V=\prod_{k=1}^{K} V_{k}, \quad V_{k}=L^{2}\left(\partial \Omega_{k}\right)^{N} \\
& x=\left(x_{k}\right), \quad x_{k}=-\sigma\left(u_{k}\right) \nu_{k}+i \omega \rho u_{k} .
\end{aligned}
$$

The operators $E, S$ and $A$ We define

$$
E \in \mathcal{L}\left(V, \prod_{k=1}^{K} H^{1}\left(\partial \Omega_{k}\right)^{N}\right)
$$

such that

$$
e=\left(e_{k}\right)=E(y)
$$

if and only if $e_{k}$ is the solution of

$$
\left\{\begin{array}{l}
-\operatorname{div} \sigma\left(e_{k}\right)-\rho \omega^{2} e_{k}=0 \quad \text { in } \Omega_{k} \\
\sigma\left(e_{k}\right) \nu+i \omega \rho e_{k}=y_{k} \quad \text { on } \partial \Omega_{k},
\end{array}\right.
$$

then

$$
S \in \mathcal{L}(V)
$$

by

$$
x=S y,
$$

if and only if

$$
\forall k \leq K, \quad x_{k}=-\sigma\left(e_{k}\right) \nu+i \omega \rho e_{k}, \quad\left(e_{k}\right) \text { solution of }(245),
$$

and finally

$$
A=S \Pi \in \mathcal{L}(V),
$$

where $\Pi$ is defined as in section 2 , except the fact it is now applied to vector fields.

\subsubsection{D Maxwell's system}

Notation In what follows

- $\Omega \subset R^{3}$ is the (bounded) propagation medium

- $\varepsilon$ is the electric permittivity of this medium

- $\mu$ is its magnetic permeability

- $\mathbf{E}=\left(\mathbf{E}_{i}\right)$ denotes the electric field (the unknown) 
The model problem For a given current density $\mathbf{j} \in L^{2}(\Omega)^{3}$, we consider the problem

$$
\left\{\begin{array}{l}
\text { Find } \mathbf{E} \in H(\operatorname{curl} ; \Omega) \text { such that } \\
\operatorname{curl}\left(\mu^{-1} \operatorname{curl} \mathbf{E}\right)-\varepsilon \omega^{2} \mathbf{E}=i \omega \mathbf{j} \quad \text { in } \Omega \\
\operatorname{curl} \mathbf{E} \times \nu+i \omega \nu \times(\mathbf{E} \times \nu)=0 \quad \text { on } \partial \Omega
\end{array}\right.
$$

Remark 13 The variational problem equivalent to (250) is not so standard. It must be set in the space

$$
\mathcal{V}(\Omega)=\left\{\mathbf{E} \in H(\operatorname{curl} ; \Omega) / \nu \times(\mathbf{E} \times \nu) \in L_{t}^{2}(\partial \Omega)\right\}
$$

where $L_{t}^{2}(\partial \Omega)$ is the space of tangential square integrable vector fields on $\partial \Omega$

$$
L_{t}^{2}(\partial \Omega)=\left\{\varphi \in L^{2}(\partial \Omega)^{3} / \varphi \cdot \nu=0\right\} .
$$

As a consequence, the solution $\mathbf{E}$ of (250) satisfies

$$
\left(\operatorname { c u r } \left[\mathbf{E} \times \nu, \nu \times(\mathbf{E} \times \nu) \in L_{t}^{2}(\partial \Omega)^{2} .\right.\right.
$$

The space $V$ and the unknown $x \quad$ In this case we have

$$
\begin{aligned}
& V=\prod_{k=1}^{K} V_{k} \quad V_{k}=L^{2}\left(\partial \Omega_{k}\right)^{N} \\
& x=\left(x_{k}\right) \quad x_{k}=-\operatorname{curl}_{k} \times \nu+i \omega \nu \times\left(\mathbf{E}_{k} \times \nu\right) .
\end{aligned}
$$

The operators $E, S$ and $A$ We define

$$
E \in \mathcal{L}\left(V, \prod_{k=1}^{K} H\left(\operatorname{curl} ; \Omega_{k}\right)\right)
$$

such that

$$
\mathbf{F}=\left(\mathbf{F}_{k}\right)=E(y),
$$

if and only if $\mathbf{F}_{k}$ is the solution of

$$
\left\{\begin{array}{lr}
\operatorname{curl}\left(\mu^{-1} \operatorname{curl} \mathbf{F}_{k}\right)-\varepsilon \omega^{2} \mathbf{F}_{k}=i \omega \mathbf{j} & \text { in } \Omega \\
\operatorname{curl} \mathbf{F}_{k} \times \nu+i \omega \nu \times\left(\mathbf{F}_{k} \times \nu\right)=0 & \text { on } \partial \Omega .
\end{array}\right.
$$

The remark we made for problem (250) also applies here. In fact

$$
E \in \mathcal{L}\left(V, \prod_{k=1}^{K} \mathcal{V}\left(\Omega_{k}\right)\right)
$$


and each $\mathbf{F}_{k}$ satisfies

$$
\operatorname{curlF}_{k} \times \nu \in L_{t}^{2}(\partial \Omega)
$$

This permits us to define

$$
S \in \mathcal{L}(V)
$$

by

$$
x=S y,
$$

if and only if

$$
\forall k \leq K, \quad x_{k}=-\operatorname{curl}_{k} \times \nu+i \omega \nu \times\left(\mathbf{F}_{k} \times \nu\right) \quad \mathbf{F}_{k} \text { solution of (254), }
$$

and finally

$$
A=S \Pi \in \mathcal{L}(V) .
$$

\section{Numerical results}

In this section, we present the results of $2 \mathrm{D}$ numerical simulations on simple test cases. The corresponding results aim at illustrating our theoretical results. In all cases, the domain $\Omega$ is a rectangle, as well as the subdomains $\Omega_{k}$. The space discretisation is obtained by the mixed finite element method described in section 6.1, except the fact we work with a uniform mesh made of small squares ( $\mathrm{cf}$ [21] for a precise description of the corresponding discrete system).

\subsection{An illustration of the interest of non local operators}

In this section, $\Omega$ is the square $]-L / 2, L / 2[\times] 0, L[$ and we consider here the two domains decomposition described in section 5 . We choose the frequency such that $\omega L=6 \pi$, i.e. the total size of the domain is 3 wavelenghts times 3 wavelenghts. The boundary conditions are the same as in section 5. Our experiment corresponds to a point source located at the center of the left subdomain.

In a first simulation, we fix the stepsize $h=0.1$ (corresponding to 10 points per wavelength), and we use the operator $T_{\alpha \beta}$ as transmission operator. We compare the convergence of the iterative algorithm when we take successively:

- $\alpha=1, \quad \beta=0 \quad$ (identity operator)

- $\alpha=1, \quad \beta=0.5 \quad$ (non local operator)

In figure 6, we plot on the same picture the variations of the normalized numerical residue as a function of the number of iterations for the two simulations. The result illustrates clearly the interest of the non local operator. One observes that the convergence is approximately the same with the two methods during the first iterations. This is due to the fact that we see essentially at the beginning the decay of the low frequency content of the error, which 


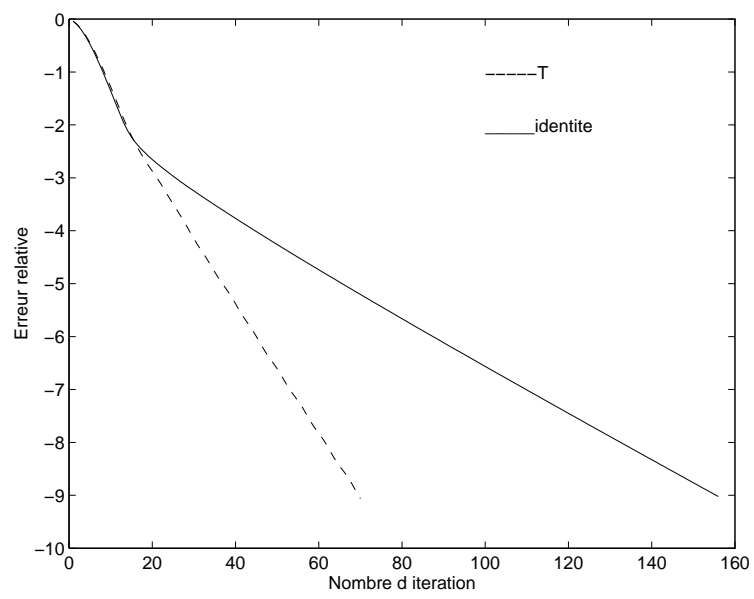

Figure 6: Logarithm of the relative error versus the number of iterations. Comparison between operator $T_{\alpha, \beta}$ and Identity operator ( 2 domains, $\omega L=6 \pi$ and $h=0.1$ )

is here dominant because the point source is located sufficiently far from the interface (It would not be the case if we had put it closer, in which case the interest of the non local operator would appear even more clearly). After a while, identity operator has difficulties with the high frequencies, which is not the case with the non local operator.

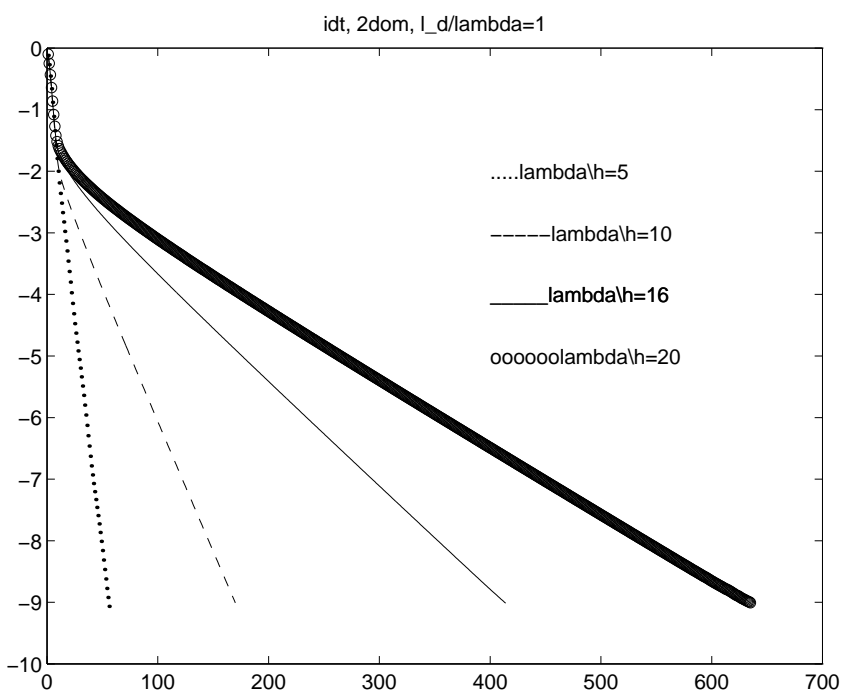

Figure 7: Logarithm of the relative error versus the number of iterations. Case of the identity Operator, The different curves correspond to different discretization step

In our second experiment, we would like to illustrate the influence of the space discretisation on the convergence of the method. Let us first remark that after discretization the problem is finite dimensional and the convergence of the method is always exponential : in other words, the operator $A^{h}$ of section 5.1 is strictly contractant. However, taking into 


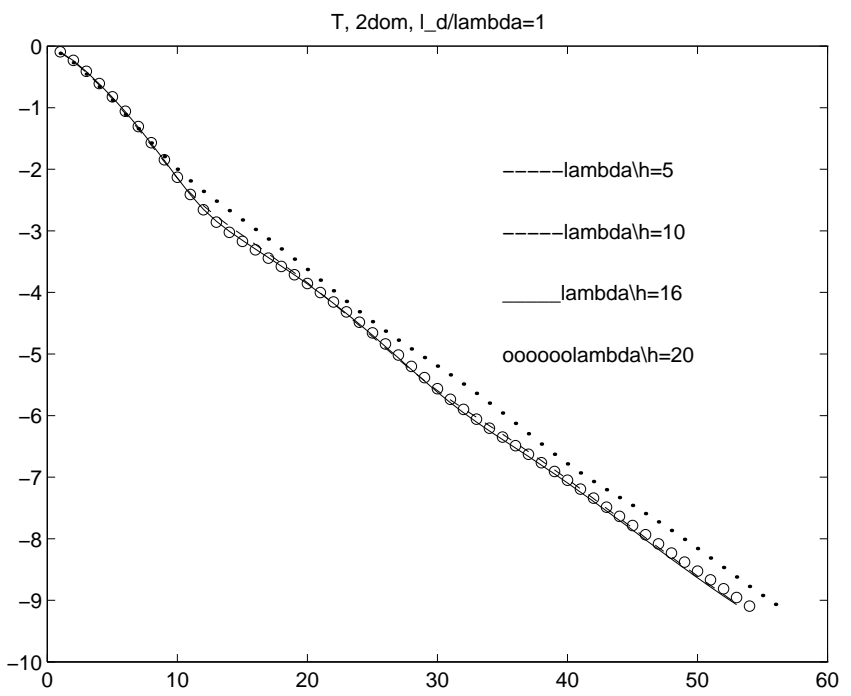

Figure 8: Logarithm of the relative error versus the number of iterations. The different curves correspond to different discretization step. Case of operator $T_{\alpha, \beta}$, variation of the discretization step

account the theoretical results relative to the continuous problem, one may expect that the rate of convergence will depend on $h$ with the identity operator but not with the non local operator.

This is confirmed by the numerical simulations. In figures 7 and 8 , we represent the variations of the normalized numerical residue as a function of the number of iterations for $h=0.2,0.1,0.0625,0.05$ respectively for the identity operator (Fig. 7) and the non local operator (Fig.8). We observe that contrary to the case of the non local operator, with the identity, diminishing $h$ deteriorates the convergence.

\subsection{A new choice : transparent like operators}

In [20], we introduced the notion of tranparent transmission operator which corresponds to define $T_{j k}$ by (178) using

$$
\alpha=1 \quad \beta=-1 .
$$

In this case, as the operator $I+\frac{1}{\omega^{2}} \Delta_{j k}$ is no longer symmetric positive definite, the square root appearing in (178) has to be carefully defined. It appears, see [20], that the good choice is the one which is consistent (think to the diagonalisation of $\Delta_{j k}$ ) with the complex square root with positive imaginary part. Of course, the operator $T_{j k}$ is no longer symmetric positive definite, and our theory can not be applied any longer: we have no guaranty of convergence for the corresponding iterative algorithms.

However, such a choice appears naturally inf one looks at a "1D like" problem : consider a domain $\Omega \subset R^{2}$ as the strip ] $-a, a[\times] 0, L[$ and let us take the same boundary conditions as in section 5 . We want to decompose in vertical strips with interfaces $x=x_{k}$ as in our 
1D model problem (cf introduction). Introducing, with the appropriate definition of the square root, the operator

$$
T=\left(I+\frac{\partial_{y y}^{2}}{\omega^{2}}\right)^{\frac{1}{2}}
$$

The Helmholtz equation writes at least formally

$$
\partial_{x x}^{2} u+(\omega T)^{2} u=f,
$$

which as the same form as the 1D Helmholtz equation of section 1, except that $\omega$ is replaced by $\omega T$. This justifies to write transmission in the form

$$
\left[\partial_{x} u+i \omega T u\right]=0
$$

and thus to use the transmission operators $T_{j k}$ introduced above.

In spite of the absence of theory in this case, we have chosen to implement these "transparent" transmission operators and to compare them with the other non local ones.

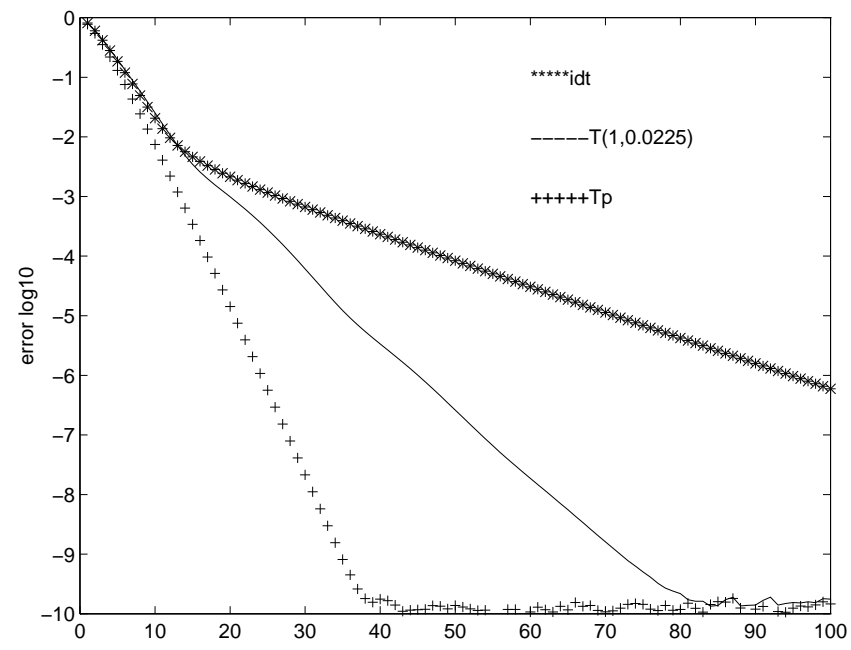

Figure 9: Logarithm of the relative error versus the number of iterations. Comparison between the transparent operator, $T_{\alpha, \beta}$ and Identity

Figure 9 demonstrates clearly the improvement provided by the transparent operator.

\subsection{On the influence of the geometry of the partition of $\Omega$}

When using the domain decomposition method, one may wonder what is the best way to build the partitionning of the domain. As a matter of fact, it is a delicate question at its answer will necessarily depend on the used computer.

We have chosen to compare a splitting in vertical layers and a second one in small squares of equal surface. We make the numerical experiments for two sizes of domains 
$\omega L=8 \pi$ and $\omega L=12 \pi$ (i.e. the initial large domain is a square of respectively 4 then 6 wavelengths). For the two splittings, we have experimented the Jacobi algorithm for different size of sub-domains (cutting in $\mathrm{Nd}=2,4, . ., 20$ sub-layers or in $\mathrm{Nd}=4,16, . ., 36$ squares) and compared the computation time required for achieving a given accuracy of $10^{-3}$ or $10^{-6}$. As a result, the best splitting appears to be a layer of one half wavelenght or a square of approximatively 1 wavelenght. Furthermore, the partitioning in square gives better performance than the partitioning in layers.

\begin{tabular}{|c||c||c||c||c|}
\hline erreur & $\omega L=8 \pi$, layers & $\omega L=8 \pi$, squares & $\omega L=12 \pi$, layers & $\omega L=12 \pi$, squares \\
\hline \hline$\epsilon=10^{-3}$ & $\mathrm{~T}=54 \mathrm{~s}, \mathrm{Nd}=8$ & $\mathrm{~T}=20 \mathrm{~s}, \mathrm{Nd}=25$ & $\mathrm{~T}=106 \mathrm{~s}, \mathrm{Nd}=12$ & $\mathrm{~T}=32 \mathrm{~s}, \mathrm{Nd}=36$ \\
\hline$\epsilon=10^{-6}$ & $\mathrm{~T}=96 \mathrm{~s}, \mathrm{Nd}=8$ & $\mathrm{~T}=34 \mathrm{~s}, \mathrm{Nd}=25$ & $\mathrm{~T}=220 \mathrm{~s}, \mathrm{Nd}=12$ & $\mathrm{~T}=70 \mathrm{~s}, \mathrm{Nd}=30$ \\
\hline
\end{tabular}

Table 3: Computational time and optimal number of sub-domains for two different sizes of problem and two different strategy of splitting

\section{References}

[1] J.D. Benamou. A massively parallel algorithm for the optimal control of systems governed by elliptic p.d.e.'s. In Seventh SIAM conference on parallel processing for scientific computing (San Francisco). SIAM, 1995.

[2] J.D. Benamou. Domain decomposition methods with coupled transmission conditions for the optimal control of systems governed by elliptic partial differential equations. SIAM J. Numer. Anal., 33:2401-2416, 1996.

[3] J.D. Benamou and B. Després. A domain decomposition method for the Helmholtz equation and related optimal control problems. J. Comp. Physics, 136:68-82, 1997.

[4] F. Brezzi and M. Fortin. Mixed and Hybrid Finite Element Methods. Springen-Verlag, 1991.

[5] X.C Cai, M.A. Casarin, F.W. Elliot, and O.B. Widlund. Overlapping schwartz algorithms for solving helmholtz's equation. In 10th international symposium on domain decomposition methods for partial differential equations (Boulder), 1997.

[6] P. Chevalier and F. Nataf. An optimized order 2 method for the helmholtz equation. In 10th international symposium on domain decomposition methods for partial differential equations (Boulder), 1997.

[7] P. Chevalier and F. Nataf. An optimized order 2 (oo2) method for the helmholtz equation. C. R. Acad. Sci. Paris Sé. I Math., (6):769-774, 1998.

[8] F. Clement, M. Kern, and C. Rubin. Solution of the 3D Helmholtz equation by conjugate gradients. In Copper mountain conference on iterative methods, 1990. 
[9] F. Collino, G. Delbue, P.Joly, and A. Piacentini. A new transmission operator in the non-overlapping domain decomposition for the maxwell equations. Comput. Methods Appl. Mech. Engrg., pages 195-207, 1997.

[10] D. Colton and R. Kress. Integral equation method in scattering. Wiley-Interscience, 1983.

[11] D. Colton and R. Kress. Inverse acoustic and electromagnetic scattering theory. Sringer-Verlag, 1992.

[12] B. Despres. Decomposition de domaine et probleme de helmholtz. (french. english summary). C. R. Acad. Sci. Paris Sé. I Math., 311(6):313-316, 1990.

[13] B. Després. Domain decomposition method and the Helmholtz problem. In G. Cohen, P. Joly, and L.Halpern, editors, First International Conference on Mathematical and Numerical Aspects of Wave Propagation (Strasbourg, Fr, 1991), pages 44-52. SIAMPhiladelphia, PA, 1991.

[14] B. Després. Methodes de decomposition de domaine pour les problemes de propagation d'ondes en regime harmonique. Le theoreme de Borg pour l'equation de Hill vectorielle. PhD thesis, Paris ix (Dauphine) France, 1991.

[15] B. Després. Domain decomposition method and the Helmholtz problem (part ii). In R. Kleinman, T. Angell, D. Colton, F. Santosa, and I. Stackgold, editors, Second international conference on mathematical and numerical aspects of wave propagation phenoma, pages 197-206. SIAM, 1993.

[16] B. Despres. Implementation of a non overlapping domain decomposition method on a cray t3d, for solving the $3 \mathrm{~d}$ harmonic maxwell's equations. In international symposium on Maxwell's equations. IMA, 1994.

[17] B. Després, P. Joly, and J. E. Roberts. A domain decomposition method for the harmonic maxwell's equations. In R. Beauwens and P. de Groen, editors, IMACS international symposium on iterative methods in linear algebra, pages 475-484. North Holland-Amsterdam, 1992.

[18] J. Douglas, F. Pereira, and J.E. Santos. A parallelizable approach to the simulation of waves in dispersive media. In G.Cohen, editor, Third international conference on mathematical and numerical wave propagation phenomena (Cannes-Mandelieu), pages 673-682. SIAM, 1995.

[19] O. Ernst and G. Golub. A domain decomposition approach to solving the helmholtz equation with a radiation boundary condition. In A. Quarteroni, J. Periaux, Y. Kuznetsov, and O. Widlund, editors, Domain Decomposition Methods in Science and Engineering, pages 177-191. AMS, 1992. 
[20] S. Ghanemi. Methodes de decomposition de domaines avec conditions de transmissions non locales pour des problemes de propagation d'ondes. $\mathrm{PhD}$ thesis, Paris ix (Dauphine) France, 1996.

[21] S. Ghanemi, F. Collino, and P.Joly. Domain decomposition method for harmonic wave equations. In G. Cohen, editor, Third international conference on mathematical and numerical wave propagation phenomena (Cannes-Mandelieu), pages 663-672. SIAM, 1995.

[22] P. Grisvard. Elliptic problems in non smooth domains. Monograph and studies in Mathematics. Pitman, 1985.

[23] P. Lax and R. Philipps. Scattering Theory. Academic Press, 1967.

[24] JL. Lions and E. Magenes. Problèmes aux limites non homogènes et applications. Dunod, 1968.

[25] P.L. Lions. On the Schwarz alternating method 1. In First international symposium on domain decomposition methods for partial differential equations. SIAM, 1988.

[26] P.L. Lions. On the Schwarz alternating method 2. In Second international symposium on domain decomposition methods for partial differential equations. SIAM, 1989.

[27] P.L. Lions. On the Schwarz alternating method 3. In T. F. Chan, R. Glowinski, J. Périaux, and O. B. Widlund, editors, Third international symposium on domain decomposition methods for partial differential equations, pages 202-223. SIAM, 1990.

[28] J.C. Nedelec. A new family of mixed finite elements in $r^{3}$. Num. Math., 50:57-81, 1986.

[29] P.A. Raviart and J.M. Thomas. A mixed finite element method for 2nd order elliptic problems. In Mathematical aspects of finite element methods (Proc. Conf., Consiglio Naz. delle Ricerche (C.N.R.), Rome), volume 606, pages 292-315. Proc. of math. aspects on the finite element method, Berlin Heidelberg New York: Springer, 1977.

[30] V.V. Shaidurov and E.I. Ogorodnikov. Some numerical method of solving Helmholtz wave equation. In First International Conference on Mathematical and Numerical Aspects of Wave Propagation (Strasbourg, Fr, 1991), pages 73-79. SIAM, 1991.

[31] B. Stupfel. A fast-domain decomposition method for the solution of electromagnetic scattering by large objects. IEEE Trans. Antennas and Propagation, 44(10):1375$1385,1996$.

[32] M.E. Taylor. Partial Differential Equations, Tome 1-2. Springer-Verlag, Berlin, 1996. 
Unit'e de recherche INRIA Lorraine, Technopôle de Nancy-Brabois, Campus scientifique,

615 rue du Jardin Botanique, BP 101, 54600 VILLERS LÈS NANCY

Unit'e de recherche INRIA Rennes, Irisa, Campus universitaire de Beaulieu, 35042 RENNES Cedex

Unit'e de recherche INRIA Rhône-Alpes, 655, avenue de l'Europe, 38330 MONTBONNOT ST MARTIN

Unit'e de recherche INRIA Rocquencourt, Domaine de Voluceau, Rocquencourt, BP 105, 78153 LE CHESNAY Cedex

Unit'e de recherche INRIA Sophia-Antipolis, 2004 route des Lucioles, BP 93, 06902 SOPHIA-ANTIPOLIS Cedex

Éditeur

INRIA, Domaine de Voluceau, Rocquencourt, BP 105, 78153 LE CHESNAY Cedex (France)

http://www.inria.fr

ISSN 0249-6399 